\title{
GENERATING SEQUENCES AND SEMIGROUPS OF VALUATIONS ON 2-DIMENSIONAL NORMAL LOCAL RINGS
}

\section{A Dissertation}

presented to

the Faculty of the Graduate School

University of Missouri

In Partial Fulfillment

of the Requirements for the Degree

Doctor of Philosophy

by

ARPAN DUTTA

Dr. Dale Cutkosky, Dissertation Supervisor

MAY 2018 
The undersigned, appointed by the Dean of the Graduate School, have examined the dissertation entitled

\section{GENERATING SEQUENCES AND SEMIGROUPS OF VALUATIONS ON 2-DIMENSIONAL NORMAL LOCAL RINGS}

presented by Arpan Dutta, a candidate for the degree of Doctor of Philosophy of Mathematics, and hereby certify that in their opinion it is worthy of acceptance.

Professor Dale Cutkosky

Professor Zhenbo Qin

Professor Calin Chindris

Professor Kannappan Palaniappan 


\section{ACKNOWLEDGEMENTS}

First of all, I wish to express sincere gratitude to my advisor, Professor Dale Cutkosky, for his vision and direction. I am extremely lucky to have the opportunity to work with him.

I would also like to thank my committee members Dr. Zhenbo Qin, Dr. Calin Chindris and Dr. Kannappan Palaniappan for their support and encouragement.

I am very grateful to MU Mathematics department's academic support staff Kyle Newell-Groshong and Gwen Gilpin. Their timely help and advices are deeply appreciated.

Finally, I would like to thank my parents. This would not have been possible without their constant help and support. 


\section{Contents}

Abstract $\quad$ iv

1 Notations 1

2 Introduction 2

$\begin{array}{lll}3 & \text { Subgroups of } U_{m} \times U_{n} & 7\end{array}$

4 Generating Sequences $\quad 12$

5 Valuation Semigroups of Invariant Subrings 14

6 Finite and Non-Finite Generation $\quad 16$

$\begin{array}{lll}7 & \text { Non-splitting } & 38\end{array}$

$\begin{array}{ll}\text { Bibliography } & 43\end{array}$

$\begin{array}{ll}\text { VITA } & 45\end{array}$ 
GENERATING SEQUENCES AND SEMIGROUPS OF VALUATIONS ON

2-DIMENSIONAL NORMAL LOCAL RINGS

Arpan Dutta

Dr. Dale Cutkosky, Dissertation Supervisor

\begin{abstract}
In this thesis we develop a method for constructing generating sequences for valuations dominating the ring of a two dimensional quotient singularity. Suppose that $K$ is an algebraically closed field of characteristic zero, $K[X, Y]$ is a polynomial ring over $K$ and $\nu$ is a rational rank 1 valuation of the field $K(X, Y)$ which dominates $K[X, Y]_{(X, Y)}$. Given a finite Abelian group $H$ acting diagonally on $K[X, Y]$, and a generating sequence of $\nu$ in $K[X, Y]$ whose members are eigenfunctions for the action of $H$, we compute a generating sequence for the invariant ring $K[X, Y]^{H}$. We use this to compute the semigroup $S^{K[X, Y]^{H}}(\nu)$ of values of elements of $K[X, Y]^{H}$. We further determine when $S^{K[X, Y]}(\nu)$ is a finitely generated $S^{K[X, Y]^{H}}(\nu)$-module.
\end{abstract}




\section{Chapter 1}

\section{Notations}

We denote the natural numbers $\{0,1,2, \cdots\}$ by $\mathbb{N}$. We denote the positive integers by $\mathbb{Z}_{>0}$ and the positive rational numbers by $\mathbb{Q}_{>0}$. If the greatest common divisor of two positive integers $a$ and $b$ is $d$, this is denoted by $(a, b)=d$. If $\left\{\gamma_{k}\right\}_{k \geqslant 0}$ is a set of rational numbers, we define $G\left(\gamma_{0}, \cdots, \gamma_{n}\right)=\sum_{k=0}^{n} \gamma_{k} \mathbb{Z}$ and $G\left(\gamma_{0}, \gamma_{1}, \cdots\right)=\sum_{k \geqslant 0} \gamma_{k} \mathbb{Z}$. Similarly we define $S\left(\gamma_{0}, \cdots, \gamma_{n}\right)=\sum_{k=0}^{n} \gamma_{k} \mathbb{N}$ and $S\left(\gamma_{0}, \gamma_{1}, \cdots\right)=\sum_{k \geqslant 0} \gamma_{k} \mathbb{N}$. If a group $G$ is generated by $g_{1}, \cdots, g_{n}$, we denote this by $G=<g_{1}, \cdots, g_{n}>$. 


\section{Chapter 2}

\section{Introduction}

Let $R$ be a local domain with maximal ideal $m_{R}$ and quotient field $L$, and $\nu$ be a valuation of $K$ which dominates $R$. Let $V_{\nu}$ be the valuation ring of $\nu$, with maximal ideal $m_{\nu}$ and $\Phi_{\nu}$ be the valuation group of $\nu$. The associated graded ring of $R$ along the valuation $\nu$, defined by Teissier in [14] and [15], is

$$
\operatorname{gr}_{\nu}(R)=\bigoplus_{\gamma \in \Phi_{\nu}} \mathcal{P}_{\gamma}(R) / \mathcal{P}_{\gamma}^{+}(R)
$$

where

$$
\mathcal{P}_{\gamma}(R)=\{f \in R \mid \nu(f) \geqslant \gamma\} \text { and } \mathcal{P}_{\gamma}^{+}(R)=\{f \in R \mid \nu(f)>\gamma\} \text {. }
$$

In general, $\operatorname{gr}_{\nu}(R)$ is not Noetherian. The valuation semigroup of $\nu$ on $R$ is

$$
S^{R}(\nu)=\{\nu(f) \mid f \in R \backslash(0)\} .
$$

If $R / m_{R}=V_{\nu} / m_{\nu}$ then $\operatorname{gr}_{\nu}(R)$ is the group algebra of $S^{R}(\nu)$ over $R / m_{R}$, so that $\operatorname{gr}_{\nu}(R)$ is completely determined by $S^{R}(\nu)$.

A generating sequence of $\nu$ in $R$ is a set of elements of $R$ whose classes in $\operatorname{gr}_{\nu}(R)$

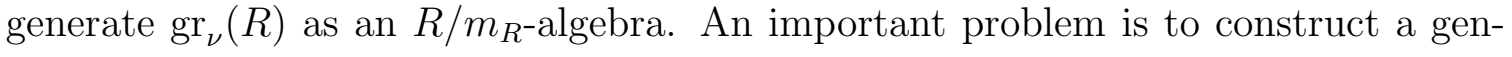
erating sequence of $\nu$ in $R$ which gives explicit formulas for the value of an arbitrary element of $R$, and gives explicit computations of the algebra (2.1) and the semigroup (2.2). For regular local rings $R$ of dimension 2, the construction of generating 
sequences is realized in a very satisfactory way by Spivakovsky [13] (with the assumption that $R / m_{R}$ is algebraically closed) and by Cutkosky and Vinh [6] for arbitrary regular local rings of dimension 2. A consequence of this theory is a simple classification of the semigroups which occur as a valuation semigroup on a regular local ring of dimension 2. There has been some success in constructing generating sequences in Noetherian local rings of dimension $\geqslant 3$, for instance in [7], [10], [11] and [15], but the general situation is very complicated and is not well understood.

Another direction is to construct generating sequences in normal 2 dimensional Noetherian local rings. This is also extremely difficult. In Section 9 of [6], a generating sequence is constructed for a rational rank 1 non discrete valuation in the ring $R=$ $k[u, v, w] /\left(u v-w^{2}\right)$, from which the semigroup is constructed. The example shows that the valuation semigroups of valuations dominating a normal two dimensional Noetherian local ring are much more complicated than those of valuations dominating a two dimensional regular local ring. In this thesis, we develop the method of this example into a general theory.

If $R$ is a 2 dimensional Noetherian local domain, and $\nu$ is a valuation of the quotient field $L$ of $R$ which dominates $R$, it follows from Abhyankar's inequality [1] that the valuation group $\Phi_{\nu}$ of $\nu$ is a finitely generated group, except in the case when the rational rank of $\nu$ is $1\left(\Phi_{\nu} \otimes \mathbb{Q} \cong \mathbb{Q}\right)$ and $\Phi_{\nu}$ is non discrete. As this is the essentially difficult case in dimension 2, we will restrict to such valuations.

Let $K$ be an algebraically closed field of characteristic 0 and $K[X, Y]$ be a polynomial ring in two variables, which has the maximal ideal $\mathfrak{m}=(x, y)$. Let $\alpha \in K$ be a primitive $m$-th root of unity and $\beta \in K$ be a primitive $n$-th root of unity. Now the 
group $\mathbb{U}_{m} \times \mathbb{U}_{n}$ acts on $K[X, Y]$ by $K$-algebra isomorphisms, where

$$
\left(\alpha^{i}, \beta^{j}\right) X=\alpha^{i} X \text { and }\left(\alpha^{i}, \beta^{j}\right) Y=\beta^{j} Y .
$$

In Theorem 3.0.2, we give a classification of the subgroups $H_{i, j, t, x}$ of $\mathbb{U}_{m} \times \mathbb{U}_{n}$. Let

$$
A_{i, j, t, x}=K[X, Y]^{H_{i, j, t, x}} \text { and } \mathfrak{n}=\mathfrak{m} \cap A_{i, j, t, x} .
$$

We say that $f \in K[X, Y]$ is an eigenfunction for the action of $H_{i, j, t, x}$ on $K[X, Y]$ if for all $g \in H_{i, j, t, x}, g f=\lambda_{g} f$ for some $\lambda_{g} \in K$.

Let $\nu$ be a rational rank 1 non discrete valuation dominating the local ring $K[X, Y]_{\mathfrak{m}}$. Using the algorithm of [13] or [6], we construct a generating sequence

$$
Q_{0}=X, Q_{1}=Y, Q_{2}, \ldots
$$

of $\nu$ in $K[X, Y]$. Let $\nu^{*}$ be the restriction of $\nu$ to the quotient field of $A_{i, j, t, x}$. In Theorem 5.0.1, we construct a generating sequence of $\nu^{*}$ in $A_{i, j, t, x}$, when the members of the generating sequence (2.3) are eigenfunctions for the action of $H_{i, j, t, x}$ on $K[X, Y]$. We give an explicit construction of the valuation semigroups $S^{\left(A_{i, j, t, x}\right)_{\mathfrak{n}}}(\nu)$ in Theorem 5.0 .1 .

Suppose that a Noetherian local domain $B$ dominates a Noetherian local domain $A$. Let $L$ be the quotient field of $A, M$ be the quotient field of $B$ and suppose that $M$ is finite over $L$. Suppose that $\omega$ is a valuation of $L$ which dominates $A$ and $\omega^{*}$ is an extension of $\nu$ to $M$ which dominates $B$. We can ask if $\operatorname{gr}_{\omega^{*}}(B)$ is a finitely generated $\operatorname{gr}_{\omega}(A)$-module or if $S^{B}\left(\omega^{*}\right)$ is a finitely generated $S^{A}(\omega)$-module. In general, $\operatorname{gr}_{\omega^{*}}(B)$ is not a finitely generated $\operatorname{gr}_{\omega}(A)$-algebra, so is certainly not a finitely generated $\operatorname{gr}_{\omega}(A)$-module. However, is is shown in Theorem 1.5. [4] that if $A$ and $B$ are essentially of finite type over a field characteristic zero, then there 
exists a birational extension $A_{1}$ of $A$ and a birational extension $B_{1}$ of $B$ such that $\omega^{*}$ dominates $B_{1}, \omega$ dominates $A_{1}, B_{1}$ dominates $A_{1}$ and $\operatorname{gr}_{\omega^{*}}\left(B_{1}\right)$ is a finitely generated $\operatorname{gr}_{\omega}\left(A_{1}\right)$-module (so $S^{B_{1}}\left(\omega^{*}\right)$ is a finitely generated $S^{A_{1}}(\omega)$-module).

The situation is much more subtle in positive characteristic and mixed characteristic. In Theorem 1 [5], it is shown that If $A$ and $B$ are excellent of dimension two and $L \rightarrow M$ is separable, then there exist birational extension $A_{1}$ of $A$ and $B_{1}$ of $B$ such that $A_{1}$ and $B_{1}$ are regular, $B_{1}$ dominates $A_{1}$, $\omega^{*}$ dominates $B_{1}$ and $\operatorname{gr}_{\omega^{*}}\left(B_{1}\right)$ is a finitely generated $\operatorname{gr}_{\omega}\left(A_{1}\right)$-algebra if and only if the valued field extension $L \rightarrow M$ is without defect. For a discussion of defect in a finite extension of valued fields, see $[8]$.

In this thesis, we completely answer the question of finite generation of $S^{\left[K[X, Y]_{\mathfrak{m}}\right.}(\nu)$ as a $S^{\left(A_{i, j, t, x}\right)_{\mathfrak{n}}}(\nu)$-module (and hence of $\operatorname{gr}_{\nu}\left(K[X, Y]_{\mathfrak{m}}\right)$ as a $\operatorname{gr}_{\nu}\left(\left(A_{i, j, t, x}\right)_{\mathfrak{n}}\right)$-module) for valuations with a generating sequence of eigenfunctions. We obtain the following results in Chapter 6.

Proposition 2.0.1. Let $R_{\mathfrak{m}}=K[X, Y]_{(X, Y)}$ and $H_{i, j, t, x}$ be a subgroup of $\mathbb{U}_{m} \times \mathbb{U}_{n}$. Let $\nu$ be a rational rank 1 non discrete valuation $\nu$ dominating $R_{\mathfrak{m}}$ with a generating sequence (2.3) of eigenfunctions for $H_{i, j, t, x}$. Then $S^{R_{\mathfrak{m}}}(\nu)$ is finitely generated over the subsemigroup $S^{\left(A_{i, j, t, x}\right)_{\mathfrak{n}}}(\nu)$ if and only if $\exists N \in \mathbb{Z}_{>0}$ such that $Q_{r} \in A_{i, j, t, x} \forall r \geqslant N$. Further, if $Q_{N} \in A_{i, j, t, x}$, then $Q_{M} \in A_{i, j, t, x} \forall M \geqslant N \geqslant 1$.

Theorem 2.0.2. Let $R_{\mathfrak{m}}=K[X, Y]_{(X, Y)}$ and $H_{i, j, t, x}$ be a subgroup of $\mathbb{U}_{m} \times \mathbb{U}_{n}$.

1) $\exists$ a rational rank 1 non discrete valuation $\nu$ dominating $R_{\mathfrak{m}}$ with a generating sequence (2.3) of eigenfunctions for $H_{i, j, t, x} \Longleftrightarrow\left(\frac{m}{i}, \frac{n}{j}\right)=t$.

2) If $\left(\frac{m}{i}, \frac{n}{j}\right)=t=1$, then $S^{R_{\mathfrak{m}}}(\nu)$ is a finitely generated $S^{\left(A_{i, j, t, x}\right)_{\mathfrak{n}}}(\nu)$-module for 
all rational rank 1 non discrete valuations $\nu$ which dominate $R_{\mathfrak{m}}$ and have a generating sequence (2.3) of eigenfunctions for $H_{i, j, t, x}$.

3) If $\left(\frac{m}{i}, \frac{n}{j}\right)=t>1$, then $S^{R_{\mathfrak{m}}}(\nu)$ is not a finitely generated $S^{\left(A_{i, j, t, x}\right)_{\mathfrak{n}}}(\nu)$-module for all rational rank 1 non discrete valuations $\nu$ which dominate $R_{\mathfrak{m}}$ and have a generating sequence (2.3) of eigenfunctions for $H_{i, j, t, x}$.

In Chapter 7, we show that for the valuations we consider, the restriction of $\nu$ to the quotient field of $A_{i, j, t, x}$ does not split in $K[X, Y]_{\mathfrak{m}}$. The failure of non splitting can be an obstruction to finite generation of $S^{\omega^{*}}(B)$ as an $S^{\omega}(A)$-module (Theorem $5[5])$, but our result shows that it is not a sufficient condition. 


\section{Chapter 3}

\section{Subgroups of $U_{m} \times U_{n}$}

Let $K$ be an algebraically closed field of characteristic zero. Let $\alpha$ be a primitive $m$-th root of unity, and $\beta$ be a primitive $n$-th root of unity, in $K$. We denote $\mathbb{U}_{m}=\langle\alpha\rangle$, and $\mathbb{U}_{n}=<\beta>$, which are multiplicative cyclic groups of orders $m$ and $n$ respectively.

Lemma 3.0.1 (Goursat). Let $A$ and $B$ be two groups. There is a bijective correspondence between subgroups $G \leqslant A \times B$, and 5-tuples $\left\{\overline{G_{1}}, G_{1}, \overline{G_{2}}, G_{2}, \theta\right\}$, where

$$
G_{1} \unlhd \overline{G_{1}} \leqslant A, G_{2} \unlhd \overline{G_{2}} \leqslant B, \theta: \frac{\overline{G_{1}}}{G_{1}} \rightarrow \frac{\overline{G_{2}}}{G_{2}} \text { is an isomorphism. }
$$

Proof. Let $\pi_{1}$ and $\pi_{2}$ denote the first and second projection maps respectively. Let $i_{1}: A \rightarrow A \times B$ and $i_{2}: B \rightarrow A \times B$ denote the inclusion maps. Given a subgroup $G$ of $A \times B$, we construct the elements of the 5 -tuple as follows,

$$
\begin{gathered}
\overline{G_{1}}=\pi_{1}(G), G_{1}=i_{1}^{-1}(G) \\
\overline{G_{2}}=\pi_{2}(G), G_{2}=i_{2}^{-1}(G) \\
\theta: \overline{G_{1}} \frac{\overline{G_{2}}}{G_{1}} \text { is defined by } \theta(\bar{a})=\bar{b}, \text { if }(a, b) \in G .
\end{gathered}
$$

By construction, $\overline{G_{1}}=\{a \in A \mid \exists b \in B$ with $(a, b) \in G\}$ and $G_{1}=\{a \in A \mid(a, 1) \in$ $G$. Let $x \in G_{1}, a \in \overline{G_{1}}$. Then $(x, 1) \in G$ and $(a, b) \in G$ for some $b \in B$ implies $(a, b)(x, 1)(a, b)^{-1} \in G \Longrightarrow a x a^{-1} \in G_{1} \Longrightarrow G_{1} \unlhd \overline{G_{1}}$. Similarly, we have $G_{2} \unlhd \overline{G_{2}}$. 
Conversely suppose we are given a 5-tuple $\left\{\overline{G_{1}}, G_{1}, \overline{G_{2}}, G_{2}, \theta\right\}$ satisfying the conditions of the theorem. Let $p: \overline{G_{1}} \times \overline{G_{2}} \rightarrow \frac{\overline{G_{1}}}{G_{1}} \times \frac{\overline{G_{2}}}{G_{2}}$ be the natural surjection. Let $G_{\theta}<\overline{\overline{G_{1}}} \times \frac{\overline{G_{2}}}{G_{2}}$ denote the graph of $\theta$. Then $G=p^{-1}\left(G_{\theta}\right)$.

Now we show the bijectivity of the correspondence. First we establish injectivity. Suppose $G \neq H$ be two subgroups of $A \times B$, such that the corresponding 5-tuples are equal, if possible. Thus, $\left\{\overline{G_{1}}, G_{1}, \overline{G_{2}}, G_{2}, \theta_{G}\right\}=\left\{\overline{H_{1}}, H_{1}, \overline{H_{2}}, H_{2}, \theta_{H}\right\}$. Now $G \neq$ $H \Longrightarrow \exists(a, b) \in G-H$, without loss of generality. But this contradicts $\theta_{G}=\theta_{H}$, since $\theta_{G}(\bar{a})=\bar{b}$, but $\theta_{H}(\bar{a}) \neq \bar{b}$. So this correspondence is injective.

Now we establish the surjectivity of the correspondence. Given a 5-tuple satisfying the conditions of the theorem, we construct a subgroup $G \leqslant A \times B$. Now, $G=$ $p^{-1}\left(G_{\theta}\right)=\left\{(g, h) \mid \bar{h}=\theta(\bar{g}), g \in \overline{G_{1}}, h \in \overline{G_{2}}\right\} . a \in \pi_{1}(G) \Longrightarrow(a, b) \in G$ for some $b \in$ $B \Longrightarrow a \in \overline{G_{1}}$. Conversely, $a \in \overline{G_{1}} \Longrightarrow \theta(\bar{a})=\bar{b}$ for some $b \in \overline{G_{2}} \Longrightarrow(a, b) \in$ $p^{-1}\left(G_{\theta}\right)=G \Longrightarrow a \in \pi_{1}(G)$. Thus we have shown $\pi_{1}(G)=\overline{G_{1}}$. Now, $a \in i_{1}^{-1}(G) \Longleftrightarrow$ $(a, 1) \in G=p^{-1}\left(G_{\theta}\right) \Longleftrightarrow p(a, 1)=(\bar{a}, \overline{1}) \in G_{\theta} \Longleftrightarrow \theta(\bar{a})=\overline{1} \Longleftrightarrow \bar{a}=\overline{1} \Longleftrightarrow a \in G_{1}$. Similarly we show, $\overline{G_{2}}=\pi_{2}(G), G_{2}=i_{2}^{-1}(G)$.

Theorem 3.0.2. Given positive integers $i, j, t, x$ satisfying the given conditions

$$
i|m, j| n, t\left|\frac{m}{i}, t\right| \frac{n}{j},(x, t)=1,1 \leqslant x \leqslant t
$$

let

$$
H_{i, j, t, x}=\left\{\left(\alpha^{a i}, \beta^{b j}\right) \mid b \equiv \operatorname{ax}(\bmod t)\right\}
$$

Then the $H_{i, j, t, x}$ are subgroups of $\mathbb{U}_{m} \times \mathbb{U}_{n}$. And given any subgroup $G$ of $\mathbb{U}_{m} \times \mathbb{U}_{n}$, there exist unique $i, j, t, x$ satisfying the above conditions such that $G=H_{i, j, t, x}$. 
Proof. We first show that the condition $b \equiv a x(\bmod t)$ is well defined under the given conditions on $i, j, t, x$. Suppose $\left(\alpha^{a_{1} i}, \beta^{b_{1} j}\right)=\left(\alpha^{a_{2} i}, \beta^{b_{2} j}\right)$, that is, $a_{1} i \equiv a_{2} i(\bmod m)$, and $b_{1} j \equiv b_{2} j(\bmod n)$. Then, $\frac{m}{i} \mid\left(a_{1}-a_{2}\right)$ and $\frac{n}{j} \mid\left(b_{1}-b_{2}\right)$. Thus, $t \mid\left(a_{1}-a_{2}\right)$ and $t \mid\left(b_{1}-b_{2}\right)$, hence $t \mid\left(b_{1}-b_{2}\right)-\left(a_{1}-a_{2}\right) x$. So, $\left[b_{1}-a_{1} x\right] \equiv\left[b_{2}-a_{2} x\right](\bmod t)$.

We now show $H_{i, j, t, x}$ is a subgroup of $\mathbb{U}_{m} \times \mathbb{U}_{n}$. Taking $a=b=0$, we have $(1,1) \in$ $H_{i, j, t, x}$. Let $\left(\alpha^{a i}, \beta^{b j}\right),\left(\alpha^{c i}, \beta^{d j}\right) \in H_{i, j, t, x}$ be distinct elements. Then $b \equiv a x(\bmod \mathrm{t})$, and $d \equiv c x(\bmod \mathrm{t})$. Hence $(b-d) \equiv(a-c) x(\bmod \mathrm{t})$. So, $\left(\alpha^{(a-c) i}, \beta^{(b-d) j}\right)=$ $\left(\alpha^{a i}, \beta^{b j}\right)\left(\alpha^{c i}, \beta^{d j}\right)^{-1} \in H_{i, j, t, x}$. Hence $H_{i, j, t, x}$ is a subgroup.

By Goursat's Lemma, the subgroups of $\mathbb{U}_{m} \times \mathbb{U}_{n}$ are in bijective correspondence with the 5-tuples $\left\{\overline{G_{1}}, G_{1}, \overline{G_{2}}, G_{2}, \theta\right\}$, where $G_{1} \unlhd \overline{G_{1}} \leqslant \mathbb{U}_{m}, G_{2} \unlhd \overline{G_{2}} \leqslant \mathbb{U}_{n}, \theta$ : $\frac{\overline{G_{1}}}{G_{1}} \simeq \frac{\overline{G_{2}}}{G_{2}}$. Now any subgroup of $\mathbb{U}_{m}=<\alpha>$ is of the form $H_{i}=<\alpha^{i}>=\mathbb{U}_{\frac{m}{i}}$, where $i \mid m$. Since $H_{i}$ is an abelian group, any subgroup is normal. Any subgroup of $H_{i}$ is of the form $H_{i t_{i}}=<\alpha^{i t_{i}}>=\mathbb{U}_{\frac{m}{i t_{i}}}$, where $t_{i} \mid \frac{m}{i}$. Similarly, any subgroup of $\mathbb{U}_{n}$ is of the form $H_{j}=<\beta^{j}>=\mathbb{U}_{\frac{n}{j}}$, where $j \mid n$. And any subgroup of $H_{j}$ is of the form $H_{j t_{j}}=<\beta^{j t_{j}}>=\mathbb{U}_{\frac{n}{j t_{j}}}$, where $t_{j} \mid \frac{n}{j}$. Now, $\frac{\mathbb{U} \frac{m}{i}}{\mathbb{U} \frac{m}{i t_{i}}} \simeq \mathbb{U}_{t_{i}}$ and $\frac{\mathbb{U}^{\frac{n}{j}}}{\mathbb{\mathbb { U }} \frac{n}{j t_{j}}} \simeq \mathbb{U}_{t_{j}}$. So,

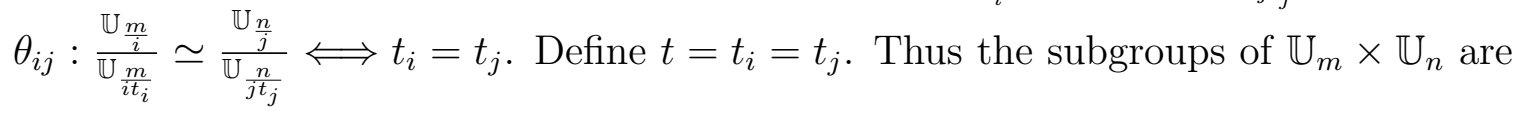
in bijective correspondence with the set of 5-tuples,

$$
\begin{aligned}
& \qquad\left(<\alpha^{i t}>,<\alpha^{i}>,<\beta^{j t}>,<\beta^{j}>, \theta_{i j}\right) \\
& \text { where } i|m, j| n, t\left|\frac{m}{i}, t\right| \frac{n}{j} \text { and } \theta_{i j}: \frac{<\alpha^{i}>}{<\alpha^{i t}>} \simeq \frac{<\beta^{j}>}{<\beta^{j t}>} \text {. }
\end{aligned}
$$

Any such isomorphism is given by $\theta_{i j}\left(\overline{\alpha^{i}}\right)=\overline{\beta^{x j}}$, where $(x, t)=1,1 \leqslant x \leqslant t$, and $\bar{v}$ denotes the residue of an element $v \in<\alpha^{i}>$ in $\frac{\left\langle\alpha^{i}\right\rangle}{\left\langle\alpha^{i t}\right\rangle}$, or the residue of an element $v \in<\beta^{j}>$ in $\frac{<\beta^{j}>}{\left.<\beta^{j t}\right\rangle}$.

If $G_{\theta_{i j}}$ denotes the graph of $\theta_{i j}$, then $G_{\theta_{i j}}=\left\{\left(\overline{\alpha^{r i}}, \overline{\beta^{r x j}}\right) \mid r \in \mathbb{N}\right\}$. Denoting the natural 
surjection $p:<\alpha^{i}>\times<\beta^{j}>\longrightarrow \frac{<\alpha^{i}>}{<\alpha^{i t}>} \times \frac{<\beta^{j}>}{<\beta^{j t}>}$, we have

$$
\begin{aligned}
p^{-1}\left(G_{\theta_{i j}}\right) & =\left\{\left(\alpha^{a i}, \beta^{b j}\right) \mid \alpha^{\overline{a i}}=\alpha^{\overline{r i}}, \beta^{\overline{b j}}=\beta^{\overline{r x j}}, \text { for some } r \in \mathbb{N}\right\} \\
& =\left\{\left(\alpha^{a i}, \beta^{b j}\right) \mid \alpha^{(a-r) i} \in<\alpha^{i t}>, \beta^{(b-r x) j} \in<\beta^{j t}>, \text { for some } r \in \mathbb{N}\right\} \\
& =\left\{\left(\alpha^{a i}, \beta^{b j}\right) \mid a \equiv r(\bmod \mathrm{t}), b \equiv r x(\bmod \mathrm{t}), \text { for some } r \in \mathbb{N}\right\} .
\end{aligned}
$$

We now show that,

$$
a \equiv r(\bmod \mathrm{t}), b \equiv r x(\bmod \mathrm{t}), \text { for some } r \in \mathbb{N} \Longleftrightarrow b \equiv a x(\bmod \mathrm{t})
$$

If $a \equiv r(\bmod \mathrm{t}), b \equiv r x(\bmod \mathrm{t})$, then $a-r=t d$ for some integer $d$. Then $b-$ $a x=b-(t d+r) x \equiv b-r x(\bmod \mathrm{t}) \equiv 0(\bmod \mathrm{t}) \Longrightarrow b \equiv a x(\bmod \mathrm{t})$. Conversely if $b \equiv a x(\bmod \mathrm{t})$, and $a \equiv r(\bmod \mathrm{t})$ for some $r$, then $b \equiv r x(\bmod \mathrm{t})$. Thus we have established (3.3). So, $p^{-1}\left(G_{\theta_{i j}}\right)=\left\{\left(\alpha^{a i}, \beta^{b j}\right) \mid b \equiv a x(\bmod \mathrm{t})\right\}$. Thus we have that any subgroup of $\mathbb{U}_{m} \times \mathbb{U}_{n}$ is of the form

$$
H_{i, j, t, x}=\left\{\left(\alpha^{a i}, \beta^{b j}\right)|b \equiv a x(\bmod \mathrm{t}) ; i| m, j|n, t| \frac{m}{i}, t \mid \frac{n}{j},(x, t)=1,1 \leqslant x \leqslant t\right\} .
$$

We now establish uniqueness. Let $\left(i_{1}, j_{1}, t_{1}, x_{1}\right)$ and $\left(i_{2}, j_{2}, t_{2}, x_{2}\right)$ be two distinct quadruples satisfying the conditions of the theorem, such that $H_{i_{1}, j_{1}, t_{1}, x_{1}}=H_{i_{2}, j_{2}, t_{2}, x_{2}}$. From (3.2), we observe $H_{i_{1}, j_{1}, t_{1}, x_{1}}=H_{i_{2}, j_{2}, t_{2}, x_{2}}$ implies

$$
\begin{aligned}
& \left(<\alpha^{i_{1} t_{1}}>,<\alpha^{i_{1}}>,<\beta^{j_{1} t_{1}}>,<\beta^{j_{1}}>, \theta_{i_{1} j_{1}}^{(1)}\right) \\
= & \left(<\alpha^{i_{2} t_{2}}>,<\alpha^{i_{2}}>,<\beta^{j_{2} t_{2}}>,<\beta^{j_{2}}>, \theta_{i_{2} j_{2}}^{(2)}\right) .
\end{aligned}
$$

Now,$<\alpha^{i_{1}}>=<\alpha^{i_{2}}>\Longrightarrow\left|<\alpha^{i_{1}}>\right|=\left|<\alpha^{i_{2}}>\right| \Longrightarrow m / i_{1}=m / i_{2} \Longrightarrow i_{1}=i_{2}=$ $i$. And, $\left\langle\alpha^{i t_{1}}>=<\alpha^{i t_{2}}>=m / i t_{1}=m / i t_{2}=t_{1}=t_{2}=t\right.$. Similarly $j=j_{1}=j_{2}$. 
Now, $\theta_{i j}^{(1)}=\theta_{i j}^{(2)} \Longrightarrow \theta_{i j}^{(1)}\left(\overline{\alpha^{i}}\right)=\theta_{i j}^{(2)}\left(\overline{\alpha^{i}}\right) \Longrightarrow \overline{\beta^{x_{1 j}}}=\overline{\beta^{x_{2} j}}$ in $\frac{<\beta^{j}>}{\left.<\beta^{t j}\right\rangle}$. Thus, $t|| x_{1}-x_{2} \mid$. Since $0<x_{1}, x_{2} \leqslant t$, we have $\left|x_{1}-x_{2}\right|=0$, i.e. $x_{1}=x_{2}$. Let $x=x_{1}=x_{2}$. Then $(i, j, t, x)=\left(i_{1}, j_{1}, t_{1}, x_{1}\right)=\left(i_{2}, j_{2}, t_{2}, x_{2}\right)$ is unique.

Proposition 3.0.3. Let $i, j, t, x$ be positive integers satisfying the conditions of Theorem 3.0.2 such that $\left(\frac{m}{i}, \frac{n}{j}\right)=t$. Write $\frac{m}{i}=M t$ and $\frac{n}{j}=N t$ where $M, N \in \mathbb{Z}_{>0}$ and $(M, N)=1$. Then $\left|H_{i, j, t, x}\right|=M N t$.

Proof. Recall, $H_{i, j, t, x}=\left\{\left(\alpha^{a i}, \beta^{b j}\right) \mid b \equiv a x(\bmod t)\right\}$. We observe, as elements of $H_{i, j, t, x},\left(\alpha^{a_{1} i}, \beta^{b_{1} j}\right)=\left(\alpha^{a_{2} i}, \beta^{b_{2} j}\right)$ if and only if $a_{1} \equiv a_{2}(\bmod M t)$ and $b_{1} \equiv b_{2}(\bmod N t)$. Thus every element of $H_{i, j, t, x}$ has an unique representation,

$$
H_{i, j, t, x}=\left\{\left(\alpha^{a i}, \beta^{b j}\right) \mid b \equiv a x(\bmod t), 0 \leqslant a<M t, 0 \leqslant b<N t\right\} .
$$

Hence there is a bijective correspondence,

$$
\begin{aligned}
H_{i, j, t, x} & \longleftrightarrow\{(a, b) \mid b \equiv a x(\bmod t), 0 \leqslant a<M t, 0 \leqslant b<N t, a, b \in \mathbb{Z}\} \\
& \longleftrightarrow\{(a, a x+\lambda t) \mid 0 \leqslant a<M t, 0 \leqslant a x+\lambda t<N t, a, \lambda \in \mathbb{Z}\} \\
& \longleftrightarrow\left\{(a, \lambda) \mid 0 \leqslant a<M t, 0 \leqslant \lambda+\frac{a x}{t}<N, a, \lambda \in \mathbb{Z}\right\} .
\end{aligned}
$$

Hence there are $M t$ possible choices for $a$. And for each choice of $a$, there are $N$ possible choices for $\lambda$. Thus $\left|H_{i, j, t, x}\right|=M N t$. 


\section{Chapter 4}

\section{Generating Sequences}

In this chapter we establish notation which will be used throughout the thesis. Let $R=K[X, Y]$ be a polynomial ring in two variables over an algebraically closed field $K$ of characteristic zero. Let $\mathfrak{m}=(X, Y)$ be the maximal ideal of $R$. Then $\mathbb{U}_{m} \times \mathbb{U}_{n}$ acts on $R$ by $K$-algebra isomorphisms satisfying

$$
\left(\alpha^{x}, \beta^{y}\right) \cdot\left(X^{r} Y^{s}\right)=\alpha^{r x} \beta^{s y} X^{r} Y^{s} .
$$

Thus, $R^{H_{i, j, t, x}}=\left\{\sum_{r, s} c_{r, s} X^{r} Y^{s} \in R \mid \alpha^{r a i} \beta^{s b j}=1 \forall r, s, \forall b \equiv a x(\bmod \mathrm{t})\right\}$.

$f \in R$ is defined to be an eigenfunction of $H_{i, j, t, x}$ if $\left(\alpha^{a i}, \beta^{b j}\right) \cdot f=\lambda_{a b} f$ for some $\lambda_{a b} \in K$, for all $\left(\alpha^{a i}, \beta^{b j}\right) \in H_{i, j, t, x}$. The eigenfunctions of $H_{i, j, t, x}$ are of the form $f=\sum_{r, s} c_{r, s} X^{r} Y^{s} \in R$ such that $\alpha^{r a i} \beta^{s b j}$ is a common constant $\forall r, s$ such that $c_{r, s} \neq$ $0, \forall b \equiv a x(\bmod \mathrm{t})$.

Let $\nu$ be a rational rank 1 non discrete valuation of $K(X, Y)$ which dominates $R_{\mathfrak{m}}$. The algorithm of Theorem 4.2 of [6] (as refined in Section (8) of [6]) produces a generating sequence

$$
Q_{0}=X, Q_{1}=Y, Q_{2}, \cdots
$$

of elements in $R$ which have the following properties. 
1) Let $\gamma_{l}=\nu\left(Q_{l}\right) \forall l \geqslant 0$ and $\overline{m_{l}}=\left[G\left(\gamma_{0}, \cdots, \gamma_{l}\right): G\left(\gamma_{0}, \cdots, \gamma_{l-1}\right)\right]=\min \{q \in$ $\left.\mathbb{Z}_{>0} \mid q \gamma_{l} \in G\left(\gamma_{0}, \cdots, \gamma_{l-1}\right)\right\} \forall l \geqslant 1$. Then $\gamma_{l+1}>\overline{m_{l}} \gamma_{l} \forall l \geqslant 1$.

2) Set $d(l)=\operatorname{deg}_{Y}\left(Q_{l}\right) \forall l \in \mathbb{Z}_{>0}$. Then, $Q_{l}=Y^{d(l)}+Q_{l}^{*}(X, Y)$, where $\operatorname{deg}_{Y}\left(Q_{l}^{*}(X, Y)\right)<$ $d(l)$. We have that, $d(1)=1, d(l)=\prod_{k=1}^{l-1} \overline{m_{k}} \forall l \geqslant 2$. In particular, $1 \leqslant l_{1} \leqslant$ $l_{2} \Longrightarrow d\left(l_{1}\right) \mid d\left(l_{2}\right)$.

3) Every $f \in R$ with $\operatorname{deg}_{Y}(f)=d$ has a unique expression

$$
f=\sum_{m=0}^{d}\left[\left(\sum_{l} b_{l, m} X^{l}\right) Q_{1}^{j_{1}(m)} \cdots Q_{r}^{j_{r}(m)}\right]
$$

where $b_{l, m} \in K, 0 \leqslant j_{l}(m)<\overline{m_{l}} \forall l \geqslant 1$, and $\operatorname{deg}_{Y}\left[Q_{1}^{j_{1}(m)} \cdots Q_{r}^{j_{r}(m)}\right]=m \forall m$. Writing $f_{m}=\left(\sum_{l} b_{l, m} X^{l}\right) Q_{1}^{j_{1}(m)} \cdots Q_{r}^{j_{r}(m)}$, we have that $\nu\left(f_{m}\right)=\nu\left(f_{n}\right) \Longleftrightarrow$ $m=n$. So, $\nu(f)=\min _{m}\left\{\nu\left(f_{m}\right)\right\}$.

4) From 3) we have that the semigroup $S^{R_{\mathfrak{m}}}(\nu)=\{\nu(f) \mid 0 \neq f \in R\}=S\left(\gamma_{l} \mid l \geqslant\right.$ $0)$.

Suppose that $\nu$ is a rational rank 1 non discrete valuation dominating $R_{\mathfrak{m}}$. We will say that $\nu$ has a generating sequence of eigenfunctions for $H_{i, j, t, x}$ if all $Q_{l}$ in the generating sequence (4.2) of Chapter 4 are eigenfunctions for $H_{i, j, t, x}$. 


\section{Chapter 5}

\section{Valuation Semigroups of Invariant Subrings}

Theorem 5.0.1. Let $i, j, t, x$ be positive integers satisfying the conditions of Theorem 3.0.2. Suppose that $\nu$ is a rational rank 1 non discrete valuation dominating $R_{\mathfrak{m}}$, where $R=K[X, Y]$, and $\mathfrak{m}=(X, Y)$. Suppose that $\nu$ has a generating sequence

$$
Q_{0}=X, Q_{1}=Y, Q_{2}, \cdots
$$

such that each $Q_{l} \in R$ is an eigenfunction for $H_{i, j, t, x}$. Let notation be as in Chapter 4. Then denoting $A_{i, j, t, x}=R^{H_{i, j, t, x}}$, and defining $\mathfrak{n}=\mathfrak{m} \cap A_{i, j, t, x}$ we have

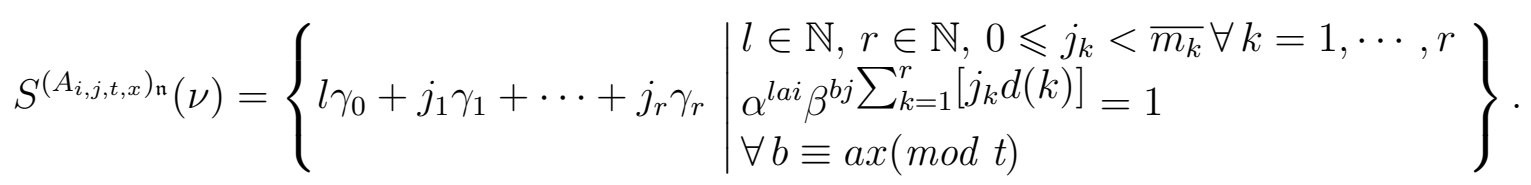

Proof. Let $0 \neq f(X, Y) \in R$, with $\operatorname{deg}_{Y}(f)=d$. By $(4.1),\left(\alpha^{a i}, \beta^{b j}\right) \cdot Y^{d(m)}=$ $\beta^{d(m) b j} Y^{d(m)}$. Since $Q_{m}$ is an eigenfunction of $H_{i, j, t, x}$, we conclude that for $m>0$,

$$
\left(\alpha^{a i}, \beta^{b j}\right) \cdot Q_{m}=\beta^{d(m) b j} Q_{m}=\beta^{\operatorname{deg}_{Y}\left(Q_{m}\right) b j} Q_{m}, \forall\left(\alpha^{a i}, \beta^{b j}\right) \in H_{i, j, t, x} .
$$

We also have, $\left(\alpha^{a i}, \beta^{b j}\right) \cdot Q_{0}=\left(\alpha^{a i}, \beta^{b j}\right) \cdot X=\alpha^{a i} X, \forall\left(\alpha^{a i}, \beta^{b j}\right) \in H_{i, j, t, x}$. Now $f$ has 
an expansion of the form 3) of Chapter 4. So,

$$
\begin{aligned}
\left(\alpha^{a i}, \beta^{b j}\right) \cdot f & =\left(\alpha^{a i}, \beta^{b j}\right) \cdot \sum_{m=0}^{d}\left[\left(\sum_{l} b_{l, m} X^{l}\right) Q_{1}^{j_{1}(m)} \cdots Q_{r}^{j_{r}(m)}\right] \\
& =\sum_{m=0}^{d}\left[\left(\sum_{l} \alpha^{l a i} b_{l, m} X^{l}\right) \beta^{b j} \sum_{k=1}^{r}\left[j_{k}(m) d(k)\right]_{\left.Q_{1}^{j_{1}(m)} \cdots Q_{r}^{j_{r}(m)}\right] .}\right.
\end{aligned}
$$

Now, $f \in A_{i, j, t, x} \Longleftrightarrow \alpha^{l a i} \beta^{b j} \sum_{k=1}^{r}\left[j_{k}(m) d(k)\right]=1, \forall b \equiv a x(\bmod \mathrm{t}), \forall l$, such that $b_{l, m} \neq 0$.

So,

$$
\begin{aligned}
& \left\{\nu(f) \mid 0 \neq f \in\left(A_{i, j, t, x}\right)_{\mathfrak{n}}\right\}=\left\{\nu(f) \mid 0 \neq f \in A_{i, j, t, x}\right\} \\
& \subset\left\{\begin{array}{l|l}
l \gamma_{0}+j_{1} \gamma_{1}+\cdots+j_{r} \gamma_{r} & \begin{array}{l}
l \in \mathbb{N}, r \in \mathbb{N}, 0 \leqslant j_{k}<\overline{m_{k}} \forall k=1, \cdots, r \\
\alpha^{l a i} \beta^{b j} \sum_{k=1}^{r}\left[j_{k} d(k)\right]=1 \\
\forall b \equiv \operatorname{ax}(\bmod \mathrm{t})
\end{array}
\end{array}\right\} .
\end{aligned}
$$

Conversely, suppose we have $l \in \mathbb{N}, r \in \mathbb{N}, 0 \leqslant j_{k}<\overline{m_{k}} \forall k=1, \cdots, r$ such that $\forall b \equiv a x(\bmod \mathrm{t})$ we have $\alpha^{l a i} \beta^{b j} \sum_{k=1}^{r}\left[j_{k} d(k)\right]=1$. Define $f(X, Y)=X^{l} Q_{1}^{j_{1}} \cdots Q_{r}^{j_{r}} \in$ $R$. For any $\left(\alpha^{a i}, \beta^{b j}\right) \in H_{i, j, t, x}$ we have, $\left(\alpha^{a i}, \beta^{b j}\right) \cdot f=\left(\alpha^{a i}, \beta^{b j}\right) \cdot\left(X^{l} Q_{1}^{j_{1}} \cdots Q_{r}^{j_{r}}\right)=$

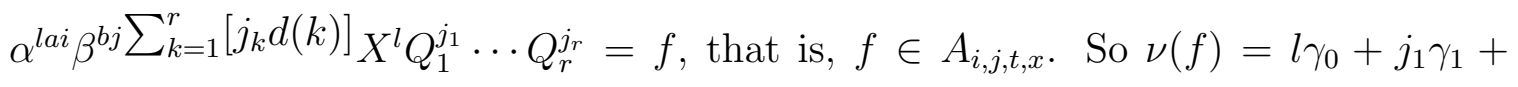
$\cdots+j_{r} \gamma_{r} \in S^{\left(A_{i, j, t, x}\right)_{\mathfrak{n}}}(\nu)$. Hence we conclude,

$$
S^{\left(A_{i, j, t, x}\right)_{\mathfrak{n}}}(\nu)=\left\{\begin{array}{l|l}
l \gamma_{0}+j_{1} \gamma_{1}+\cdots+j_{r} \gamma_{r} & \begin{array}{l}
l \in \mathbb{N}, r \in \mathbb{N}, 0 \leqslant j_{k}<\overline{m_{k}} \forall k=1, \cdots, r \\
\alpha^{l a i} \beta_{j} \sum_{k=1}^{r}\left[j_{k} d(k)\right]=1 \\
\forall b \equiv a x(\bmod \mathrm{t})
\end{array}
\end{array}\right\} .
$$




\section{Chapter 6}

\section{Finite and Non-Finite Generation}

In this chapter we study the finite and non-finite generation of the valuation semigroup $S^{R_{\mathfrak{m}}}(\nu)$ over the subsemigroup $S^{\left(A_{i, j, t, x}\right)_{\mathfrak{n}}}(\nu)$. A semigroup $S$ is said to be finitely generated over a subsemigroup $T$ if there are finitely many elements $s_{1}, \cdots, s_{n}$ in $S$ such that $S=\left\{s_{1}, \cdots, s_{n}\right\}+T$.

At the end of this chapter we will prove the following theorem.

Theorem 6.0.1. Let $R_{\mathfrak{m}}=K[X, Y]_{(X, Y)}$ and $H_{i, j, t, x}$ be a subgroup of $\mathbb{U}_{m} \times \mathbb{U}_{n}$.

1) $\exists$ a rational rank 1 non discrete valuation $\nu$ dominating $R_{\mathfrak{m}}$ with a generating sequence (4.2) of eigenfunctions for $H_{i, j, t, x} \Longleftrightarrow\left(\frac{m}{i}, \frac{n}{j}\right)=t$.

2) If $\left(\frac{m}{i}, \frac{n}{j}\right)=t=1$, then $S^{R_{\mathfrak{m}}}(\nu)$ is a finitely generated $S^{\left(A_{i, j, t, x}\right)_{\mathfrak{n}}}(\nu)$-module for all rational rank 1 non discrete valuations $\nu$ which dominate $R_{\mathfrak{m}}$ and have a generating sequence (4.2) of eigenfunctions for $H_{i, j, t, x}$.

3) If $\left(\frac{m}{i}, \frac{n}{j}\right)=t>1$, then $S^{R_{\mathfrak{m}}}(\nu)$ is not a finitely generated $S^{\left(A_{i, j, t, x}\right)_{\mathfrak{n}}}(\nu)$-module for all rational rank 1 non discrete valuations $\nu$ which dominate $R_{\mathfrak{m}}$ and have a generating sequence (4.2) of eigenfunctions for $H_{i, j, t, x}$.

We first introduce some notation. Let $\sigma(0)=0$ and for all $l \geqslant 1, \sigma(l)=$ $\min \left\{j \mid j>\sigma(l-1)\right.$ and $\left.\overline{m_{j}}>1\right\}$. Let $P_{l}=Q_{\sigma(l)}$ and $\beta_{l}=\nu\left(P_{l}\right)=\gamma_{\sigma(l)} \forall l \geqslant 0$. Let 
$\overline{n_{l}}=\left[G\left(\beta_{0}, \cdots, \beta_{l}\right): G\left(\beta_{0}, \cdots, \beta_{l-1}\right)\right]=\min \left\{q \in \mathbb{Z}_{>0} \mid q \beta_{l} \in G\left(\beta_{0}, \cdots, \beta_{l-1}\right)\right\} \forall l \geqslant 1$. Then $\overline{n_{l}}=\overline{m_{\sigma(l)}}$. $S^{R_{\mathfrak{m}}}(\nu)=S\left(\gamma_{0}, \gamma_{1}, \cdots\right)=S\left(\beta_{0}, \beta_{1}, \cdots\right)$ and $\left\{\beta_{l}\right\}_{l \geqslant 0}$ form a minimal generating set of $S^{R_{\mathfrak{m}}}(\nu)$, that is, $\overline{n_{l}}>1 \forall l \geqslant 1$.

We first make a general observation. Suppose for some $d \geqslant 1, j_{r} \neq 0$ and $l, j_{1}, \cdots, j_{r} \in \mathbb{N}$, we have an expression of the form, $\beta_{d}=l \beta_{0}+j_{1} \beta_{1}+\cdots+j_{r} \beta_{r}$. If $r>d$ then $j_{r} \beta_{r} \geqslant \beta_{r}>\beta_{d}$ which is a contradiction. If $r<d$ then $\beta_{d} \in G\left(\beta_{0}, \cdots, \beta_{d-1}\right) \Longrightarrow$ $\overline{n_{d}}=1$. This is a contradiction as $\overline{n_{l}}>1 \forall l \geqslant 1$. Thus, $\beta_{r}=l \beta_{0}+j_{1} \beta_{1}+\cdots+j_{r} \beta_{r}$. If $j_{r}>1$, then $j_{r} \beta_{r}>\beta_{r}$. If $j_{r}=0$, then $\beta_{r} \in G\left(\beta_{0}, \cdots, \beta_{r-1}\right) \Longrightarrow \overline{n_{r}}=1$. So, $j_{r}=1$. Since $\beta_{i}>0 \forall i$, we then have $l=0, j_{i}=0 \forall i \neq r$. Thus, for $l, j_{1}, \cdots, j_{r} \in \mathbb{N}$ and $d \geqslant 1$

$$
\beta_{d}=l \beta_{0}+j_{1} \beta_{1}+\cdots+j_{r} \beta_{r} \Longrightarrow j_{d}=1, l=0, j_{i}=0 \forall i \neq d
$$

Proposition 6.0.2. Let $R_{\mathfrak{m}}=K[X, Y]_{(X, Y)}$ and $H_{i, j, t, x}$ be a subgroup of $\mathbb{U}_{m} \times \mathbb{U}_{n}$. Let assumptions be as in Theorem 5.0.1. Then $S^{R_{\mathfrak{m}}}(\nu)$ is finitely generated over the subsemigroup $S^{\left(A_{i, j, t, x}\right)_{\mathfrak{n}}}(\nu)$ if and only if $\exists N \in \mathbb{Z}_{>0}$ such that $Q_{r} \in A_{i, j, t, x} \forall r \geqslant N$. Further, if $Q_{N} \in A_{i, j, t, x}$, then $Q_{M} \in A_{i, j, t, x} \forall M \geqslant N \geqslant 1$.

Proof. We first show that, for any $r \geqslant 1, \gamma_{r} \in S^{\left(A_{i, j, t, x}\right)_{\mathfrak{n}}}(\nu) \Longleftrightarrow Q_{r} \in A_{i, j, t, x}$. It is enough to show the implication $\gamma_{r} \in S^{\left(A_{i, j, t, x}\right) \mathfrak{n}}(\nu) \Longrightarrow Q_{r} \in A_{i, j, t, x}$. From (5.1) we have, $\gamma_{r} \in S^{\left(A_{i, j, t, x}\right)_{\mathfrak{n}}}(\nu) \Longrightarrow \gamma_{r}=l \gamma_{0}+j_{1} \gamma_{1}+\cdots+j_{s} \gamma_{s}$, where $l \in \mathbb{N}, s \in \mathbb{N}$, $0 \leqslant j_{k}<\overline{m_{k}}$ and $\alpha^{l a i} \beta^{b j \sum_{k=1}^{s} j_{k} d(k)}=1 \forall b \equiv a x(\bmod t)$

Since $l, j_{1}, \cdots, j_{s} \in \mathbb{N}, \gamma_{i}<\gamma_{i+1} \forall i \geqslant 1$ and $\gamma_{i}>0 \forall i$, we have $r \geqslant s$. If $r=s$, then $\gamma_{r}=l \gamma_{0}+\sum_{k=1}^{r} j_{k} \gamma_{k} \geqslant j_{r} \gamma_{r} \geqslant \gamma_{r}$. Since $j_{r} \neq 0$ and $j_{r} \in \mathbb{N}$ we have $j_{r}=1$. And $\gamma_{i}>0 \forall i$ implies $l=j_{1}=\cdots=j_{r-1}=0$. Then $\beta^{b j d(r)}=1 \forall b \equiv a x(\bmod t)$. So from (5.2), $\left(\alpha^{a i}, \beta^{b j}\right) \cdot Q_{r}=Q_{r} \forall b \equiv a x(\bmod t)$, that is, $Q_{r} \in A_{i, j, t, x}$. 
If $r>s$, then $\gamma_{r}=l \gamma_{0}+\sum_{k=1}^{s} j_{k} \gamma_{k} \Longrightarrow \overline{m_{r}}=1$. Since $0 \leqslant j_{k}<\overline{m_{k}}$, by Equation (8) in [6] we have $Q_{r+1}=Q_{r}-\lambda X^{l} Y^{j_{1}} Q_{2}^{j_{2}} \cdots Q_{s}^{j_{s}}$ where $\lambda \in K \backslash\{0\}$. Since each $Q_{m}$ is an eigenfunction for $H_{i, j, t, x}$, from (5.2) we have, $\forall b \equiv a x(\bmod t)$,

$$
\beta^{b j d(r+1)} Q_{r+1}=\beta^{b j d(r)} Q_{r}-\lambda \alpha^{l a i} \beta^{b j \sum_{k=1}^{s} j_{k} d(k)} X^{l} Y^{j_{1}} Q_{2}^{j_{2}} \cdots Q_{s}^{j_{s}} .
$$

Again by 2) in Chapter 4 we have $d(r+1)=\overline{m_{1}} \cdots \overline{m_{r}}=\overline{m_{1}} \cdots \overline{m_{r-1}}=d(r)$, as $\overline{m_{r}}=1$. So, $\beta^{b j d(r)} Q_{r+1}=\beta^{b j d(r)} Q_{r}-\lambda \alpha^{l a i} \beta^{b j \sum_{k=1}^{s} j_{k} d(k)} X^{l} Y^{j_{1}} Q_{2}^{j_{2}} \cdots Q_{s}^{j_{s}}$ for all $b \equiv a x(\bmod t)$. Since $Q_{r+1}$ is an eigenfunction, this implies $\beta^{b j d(r)}=\alpha^{l a i} \beta^{b j \sum_{k=1}^{s} j_{k} d(k)}$ $=1 \forall b \equiv a x(\bmod t)$. From (5.2), we then have $Q_{r} \in A_{i, j, t, x}$.

To prove the proposition, we now show $S^{R_{\mathrm{m}}}(\nu)$ is finitely generated over the subsemigroup $S^{\left(A_{i, j, t, x}\right)_{\mathfrak{n}}}(\nu)$ if and only if $\exists N \in \mathbb{Z}_{>0}$ such that $\forall r \geqslant N, \gamma_{r} \in S^{\left(A_{i, j, t, x}\right) \mathfrak{n}}(\nu)$. Suppose $S^{R_{\mathfrak{m}}}(\nu)$ is finitely generated over $S^{\left(A_{i, j, t, x}\right)_{\mathfrak{n}}}(\nu)$. So, $\exists x_{0}, \cdots, x_{l} \in S^{R_{\mathfrak{m}}}(\nu)$ such that $S^{R_{\mathfrak{m}}}(\nu)=\left\{x_{0}, \cdots, x_{l}\right\}+S^{\left(A_{i, j, t, x}\right)_{\mathfrak{n}}}(\nu)$. Let $L \in \mathbb{N}$ be the least natural number such that $S^{R_{\mathfrak{m}}}(\nu)=S\left(\beta_{0}, \cdots, \beta_{L}\right)+S^{\left(A_{i, j, t, x}\right)_{\mathfrak{n}}}(\nu)$, where $\beta_{i}=\gamma_{\sigma(i)} \forall i \geqslant 0$. Suppose, if possible, $\exists r>\sigma(L) \geqslant 0$ such that $\gamma_{r} \notin S^{\left(A_{i, j, t, x}\right)_{\mathfrak{n}}}(\nu)$. Choose $M$ such that $\sigma(M) \leqslant$ $r<\sigma(M+1)$. Then $\sigma(L)<\sigma(M)$, that is $L<M$. So $\beta_{L}<\beta_{M} \leqslant \gamma_{r}<\beta_{M+1}$. Now $\beta_{M}$ has an expression $\beta_{M}=\sum_{i=0}^{L} a_{i} \beta_{i}+y$ where $y \in S^{\left(A_{i, j, t, x}\right)_{\mathfrak{n}}}(\nu), a_{i} \in \mathbb{N}$. From (5.1) we have $\beta_{M}=\sum_{i=0}^{L} a_{i} \beta_{i}+\left(l \gamma_{0}+j_{1} \gamma_{1}+\cdots+j_{s} \gamma_{s}\right)$, where $0 \leqslant j_{k}<\overline{m_{k}}$ and $\alpha^{l a i} \beta^{b j \sum_{k=1}^{s} j_{k} d(k)}=1 \forall b \equiv a x(\bmod t)$. We observe $\overline{m_{k}}=1 \Longrightarrow j_{k}=0$. Thus the above expression can be rewritten as,

$$
\beta_{M}=\sum_{i=0}^{L} a_{i} \beta_{i}+\left(l \beta_{0}+j_{1} \beta_{1}+\cdots+j_{p} \beta_{p}\right)
$$

where $0 \leqslant j_{k}<\overline{n_{k}}$ and $\alpha^{l a i} \beta^{b j \sum_{k=1}^{p} j_{k} \operatorname{deg}_{Y}\left(P_{k}\right)}=1 \forall b \equiv a x(\bmod t)$. Since $L<M$, from (6.1) we obtain $j_{M}=1, a_{i}=0 \forall i=0, \cdots, L$ and $j_{k}=0 \forall k \neq M$. Thus 
$\beta^{b j \operatorname{deg}_{Y}\left(P_{M}\right)}=1 \forall b \equiv a x(\bmod t)$. From 2$)$ in Chapter 4 we have $d(r)=\overline{m_{1}} \cdots \overline{m_{r-1}}$. And, $\operatorname{deg}_{Y}\left(P_{M}\right)=d(\sigma(M))=\overline{m_{1}} \cdots \overline{m_{\sigma(M)-1}}$. Since $r \geqslant \sigma(M) \Longrightarrow r-1 \geqslant \sigma(M)-1$, we thus have $\operatorname{deg}_{Y}\left(P_{M}\right) \mid d(r)$. So, $\beta^{b j d(r)}=1 \forall b \equiv a x(\bmod t)$. From (5.2) we then conclude, $Q_{r} \in A_{i, j, t, x}$. But this contradicts $\gamma_{r} \notin S^{\left(A_{i, j, t, x}\right)_{\mathrm{n}}}(\nu)$. So, $Q_{r} \in A_{i, j, t, x} \forall r>$ $\sigma(L) \geqslant 0$, that is, $Q_{r} \in A_{i, j, t, x} \forall r \geqslant N$ for some $N \in \mathbb{Z}_{>0}$.

Conversely, we assume $S\left(\gamma_{N}, \gamma_{N+1}, \cdots\right) \subset S^{\left(A_{i, j, t, x}\right)_{\mathfrak{n}}}(\nu)$ for some $N \in \mathbb{Z}_{>0}$. Now $\gamma_{i} \in \mathbb{Q}_{>0} \forall i$ implies $\forall i \neq j, \exists d_{i}, d_{j} \in \mathbb{Z}_{>0}$ such that $d_{i} \gamma_{i}=d_{j} \gamma_{j}$. We thus have $d_{i} \gamma_{i}=d_{i, N} \gamma_{N} \forall i=0, \cdots, N-1$. We will now show that, $S^{R_{\mathfrak{m}}}(\nu)=T+S^{\left(A_{i, j, t, x}\right)_{\mathfrak{n}}}(\nu)$, where $T=\left\{\sum_{i=0}^{N-1} \overline{a_{i}} \gamma_{i} \mid 0 \leqslant \overline{a_{i}}<d_{i}\right\}$. Now, $\gamma_{i} \in S^{R_{\mathfrak{m}}}(\nu) \forall i=0, \cdots, N-1 \Longrightarrow$ $T+S^{\left(A_{i, j, t, x}\right)_{\mathfrak{n}}}(\nu) \subset S^{R_{\mathfrak{m}}}(\nu)$. So it is enough to show $S^{R_{\mathfrak{m}}}(\nu) \subset T+S^{\left(A_{i, j, t, x}\right)_{\mathfrak{n}}}(\nu)$.

$$
\begin{aligned}
& x \in S^{R_{\mathrm{m}}}(\nu) \Longrightarrow x=\sum_{i=0}^{N-1} a_{i} \gamma_{i}+\sum_{i=N}^{l} a_{i} \gamma_{i} \\
& \Longrightarrow x=\sum_{i=0}^{N-1} \overline{a_{i}} \gamma_{i}+\sum_{i=0}^{N-1} b_{i} d_{i} \gamma_{i}+\sum_{i=N}^{l} a_{i} \gamma_{i} \text { where } a_{i}=\overline{a_{i}}+b_{i} d_{i}, 0 \leqslant \overline{a_{i}}<d_{i}, b_{i} \in \mathbb{N} \\
& \Longrightarrow x=\sum_{i=0}^{N-1} \overline{a_{i}} \gamma_{i}+\sum_{i=0}^{N-1} b_{i} d_{i, N} \gamma_{N}+\sum_{i=N}^{l} a_{i} \gamma_{i} \\
& \Longrightarrow x=\sum_{i=0}^{N-1} \overline{a_{i}} \gamma_{i}+y, \text { where } y \in S^{\left(A_{i, j, t, x}\right)}(\nu) .
\end{aligned}
$$

Thus we have shown $S^{R_{\mathfrak{m}}}(\nu) \subset T+S^{\left(A_{i, j, t, x}\right)_{\mathfrak{n}}}(\nu)$. Since $T$ is a finite set, we have $S^{R_{\mathfrak{m}}}(\nu)$ is finitely generated over $S^{\left(A_{i, j, t, x}\right) \mathfrak{n}}(\nu)$.

From $(5.2),\left(\alpha^{a i}, \beta^{b j}\right) \cdot Q_{N}=\beta^{d(N) b j} Q_{N} \forall b \equiv a x(\bmod t)$. So, $Q_{N} \in A_{i, j, t, x} \Longleftrightarrow$ $\beta^{d(N) b j}=1 \forall b \equiv a x(\bmod t)$. Again from 2$)$ of Chapter 4 we have $d(N) \mid d(M) \forall M \geqslant$ $N \geqslant 1$. Hence we obtain, $Q_{N} \in A_{i, j, t, x} \Longrightarrow Q_{M} \in A_{i, j, t, x} \forall M \geqslant N \geqslant 1$. So, $S^{R_{\mathrm{m}}}(\nu)$ is not finitely generated over $S^{\left(A_{i, j, t, x}\right)_{\mathfrak{n}}}(\nu)$ if and only if $Q_{r} \notin A_{i, j, t, x} \forall r \geqslant 1$. 
Lemma 6.0.3. Let $i, j, t, x$ be positive integers satisfying the conditions of Theorem 3.0.2. Let assumptions be as in Theorem 5.0.1. Then $S^{R_{\mathfrak{m}}}(\nu)$ is not finitely generated over $S^{\left(A_{i, j, t, x}\right)_{\mathfrak{n}}}(\nu)$ if and only if $j \neq n$ and $\frac{n}{j} \nmid d(l) \forall l \geqslant 2$.

Proof. Suppose that $S^{R_{\mathfrak{m}}}(\nu)$ is not finitely generated over $S^{\left(A_{i, j, t, x}\right)_{\mathfrak{n}}}(\nu)$. Then $Q_{l} \notin$ $A_{i, j, t, x} \forall l \geqslant 1$. From (5.2), if $j=n$, then $\left(\alpha^{a i}, \beta^{b n}\right) \cdot Q_{l}=\beta^{d(l) b n} Q_{l}=Q_{l}$, that is $Q_{l} \in A_{i, n, t, x}$, which is a contradiction. So $j \neq n$. And, for some $l \geqslant 2, \frac{n}{j} \mid d(l) \Longrightarrow$ $n \mid d(l) j$. Then, $\left(\alpha^{a i}, \beta^{b j}\right) \cdot Q_{l}=\beta^{d(l) b j} Q_{l}=Q_{l}$, that is $Q_{l} \in A_{i, j, t, x}$, which is again a contradiction. So, $\frac{n}{j} \nmid d(l) \forall l \geqslant 2$.

Conversely, suppose $j \neq n$ and $\frac{n}{j} \nmid d(l) \forall l \geqslant 2$, that is, $\frac{n}{j} \nmid d(l) \forall l \geqslant 1$. Now, $(x, t)=1 \Longrightarrow a x \equiv 1(\bmod t)$ for some $a \in \mathbb{Z}$, so, $\left(\alpha^{a i}, \beta^{j}\right) \in H_{i, j, t, x}$. From $(5.2)$, $\left(\alpha^{a i}, \beta^{j}\right) \cdot Q_{l}=\beta^{d(l) j} Q_{l} \neq Q_{l}$ for all $l \geqslant 1$, as $n \nmid d(l) j$. So we have $Q_{l} \notin A_{i, j, t, x} \forall l \geqslant 1$. Hence $S^{R_{\mathfrak{m}}}(\nu)$ is not finitely generated over $S^{\left(A_{i, j, t, x}\right)_{\mathfrak{n}}}(\nu)$.

Proposition 6.0.4. Let $i, j, t, x$ be positive integers satisfying the conditions of Theorem 3.0.2, such that $\left(\frac{m}{i}, \frac{n}{j}\right)>t \geqslant 1$. Suppose that $\nu$ is a rational rank 1 non discrete valuation dominating $R_{\mathfrak{m}}$, with a generating sequence (4.2) $\left\{Q_{l}\right\}_{l \geqslant 0}$, where $Q_{0}=X, Q_{1}=Y$ as in Chapter 4. Then $\left\{Q_{l}\right\}_{l \geqslant 0}$ is not a sequence of eigenfunctions for $H_{i, j, t, x}$.

Proof. Let $d=\left(\frac{m}{i}, \frac{n}{j}\right)$. Then $1 \leqslant t<d \leqslant \min \left\{\frac{m}{i}, \frac{n}{j}\right\}$. So, $t<\frac{m}{i}$ and $t<\frac{n}{j}$. We recall, $H_{i, j, t, x}=\left\{\left(\alpha^{a i}, \beta^{b j}\right) \mid b \equiv a x(\bmod t)\right\}$. Thus $\left(\alpha^{t i}, 1\right),\left(1, \beta^{t j}\right) \in H_{i, j, t, x}$. Let $\left\{Q_{l}\right\}_{l \geqslant 0}$ be the generating sequence (4.2) with $Q_{0}=X, Q_{1}=Y$. Let $\nu\left(Q_{l}\right)=\gamma_{l} \forall l \geqslant$ 0. By Equation (8) in [6], $Q_{2}=Y^{s}-\lambda X^{r}$, where $\lambda \in K \backslash\{0\}, s \gamma_{1}=r \gamma_{0}$, and $s=$ 
$\min \left\{q \in \mathbb{Z}_{>0} \mid q \gamma_{1} \in \gamma_{0} \mathbb{Z}\right\}$. From (4.1), we have,

$$
\begin{aligned}
& \left(\alpha^{t i}, 1\right) \cdot Q_{2}=\left(\alpha^{t i}, 1\right) \cdot\left[Y^{s}-\lambda X^{r}\right]=Y^{s}-\lambda \alpha^{r t i} X^{r} . \\
& \left(1, \beta^{t j}\right) \cdot Q_{2}=\left(1, \beta^{t j}\right) \cdot\left[Y^{s}-\lambda X^{r}\right]=\beta^{s t j} Y^{s}-\lambda X^{r} .
\end{aligned}
$$

If $Q_{2}$ was an eigenfunction of $H_{i, j, t, x}$, then $m \mid r t i \Longrightarrow r=r_{1} \frac{m}{t i}$, where $r_{1} \in \mathbb{Z}_{>0}$. Similarly, $n \mid s t j \Longrightarrow s=s_{1} \frac{n}{t j}$, where $s_{1} \in \mathbb{Z}_{>0}$. And, $s \gamma_{1}=r \gamma_{0} \Longrightarrow s_{1} \frac{n}{t j} \gamma_{1}=r_{1} \frac{m}{t i} \gamma_{0}$. So, $s_{1} \frac{n}{d j} \gamma_{1}=r_{1} \frac{m}{d i} \gamma_{0}$. Now, $d \mid \frac{n}{j}$ implies $s_{1} \frac{n}{d j} \in \mathbb{Z}_{>0}$. Similarly, $r_{1} \frac{m}{d i} \in \mathbb{Z}_{>0}$. Thus, $s_{1} \frac{n}{d j} \gamma_{1} \in \gamma_{0} \mathbb{Z}$. But $t<d$ implies $s_{1} \frac{n}{d j}<s_{1} \frac{n}{t j}=s$, and this contradicts the minimality of $s$. Thus $Q_{2}$ is not an eigenfunction of $H_{i, j, t, x}$. So, $\left\{Q_{l}\right\}_{l \geqslant 0}$ is not a generating sequence of eigenfunctions for $H_{i, j, t, x}$.

We know, if $\omega$ is a primitive $l$-th root of unity in $K$, then $\left\{\omega^{k} \mid 1 \leqslant k \leqslant l\right\}$ is a complete list of all $l$-th roots of unity in $K$, and $\left\{\omega^{k} \mid 1 \leqslant k \leqslant l\right.$ and $\left.(k, l)=1\right\}$ is a complete list of all primitive $l$-th roots of unity in $K$.

We have, $\alpha$ is a primitive $m$-th root of unity and $\beta$ is a primitive $n$-th root of unity in K. Let $\delta$ be a primitive $m n$-th root of unity in $K$. Then $\delta^{n}$ is a primitive $m$-th root of unity. Now, $S_{\alpha}=\left\{\alpha^{k} \mid 1 \leqslant k \leqslant m\right.$ and $\left.(k, m)=1\right\}$ is a complete list of all primitive $m$-th roots of unity in $K$. And, $S_{\delta^{n}}=\left\{\delta^{k n} \mid 1 \leqslant k \leqslant m\right.$ and $\left.(k, m)=1\right\}$ is also a complete list of all primitive $m$-th roots of unity. Thus, $\alpha=\delta^{w_{1} n}$ where $\left(w_{1}, m\right)=$ 1 and $1 \leqslant w_{1} \leqslant m$. Similarly, $\beta=\delta^{w_{2} m}$ where $\left(w_{2}, n\right)=1$ and $1 \leqslant w_{2} \leqslant n$.

Remark 6.0.5. Let $p, q \in \mathbb{Z}$. With the notation introduced above, $\beta^{p}=\alpha^{q} \Longleftrightarrow$ $\frac{p w_{2}}{n}-\frac{q w_{1}}{m} \in \mathbb{Z}$

Proof. We have, $\beta=\delta^{w_{2} m}$ and $\alpha=\delta^{w_{1} n}$, where $\delta$ is a primitive $m n$-th root of unity. 
Thus, $\beta^{p}=\alpha^{q} \Longleftrightarrow \delta^{w_{2} m p}=\delta^{w_{1} n q} \Longleftrightarrow m n \mid\left(w_{2} m p-w_{1} n q\right) \Longleftrightarrow \frac{p w_{2}}{n}-\frac{q w_{1}}{m} \in \mathbb{Z}$.

Proposition 6.0.6. Let $i, j, t, x$ be positive integers satisfying the conditions of Theorem 3.0.2, such that $\left(\frac{m}{i}, \frac{n}{j}\right)=t, t>1$. Set $\frac{m}{i}=M t$, and $\frac{n}{j}=N t$, where $M, N \in \mathbb{Z}_{>0}$ and $(M, N)=1$. Suppose that $\exists$ a prime number $p$ such that $p \mid t$ but $p \nmid N$. Suppose that $\nu$ is a rational rank 1 non discrete valuation dominating $R_{\mathfrak{m}}$ with a generating sequence (4.2) of eigenfunctions for $H_{i, j, t, x}$. Then $S^{R_{\mathfrak{m}}}(\nu)$ is not finitely generated over $S^{\left(A_{i, j, t, x}\right)_{\mathfrak{n}}}(\nu)$.

Proof. Let $\left\{Q_{l}\right\}_{l \geqslant 0}$ be the generating sequence (4.2) of the valuation $\nu$, where $Q_{0}=$ $X, Q_{1}=Y$, and each $Q_{l}$ is an eigenfunction for $H_{i, j, t, x}$. Let $\gamma_{l}=\nu\left(Q_{l}\right) \forall l \geqslant 0$. Without any loss of generality, we can assume $\gamma_{0}=1$. Since $\nu$ is a rational valuation, we can write $\gamma_{k}=\frac{a_{k}}{b_{k}} \forall k \geqslant 1$, where $\left(a_{k}, b_{k}\right)=1$. We have, $p \mid t$, and $p \nmid N$ for a prime $p$. So $(p, N)=1$. So $\exists N_{1} \in \mathbb{Z}$ such that $N N_{1} \equiv 1(\bmod p)$. Let $w_{1}$ and $w_{2}$ be as in Remark 6.0.5. Now $\left(m, w_{1}\right)=1$ and $t \mid m$. So $\left(t, w_{1}\right)=1$. So $\left(p, w_{1}\right)=1$. So $\exists \overline{w_{1}} \in \mathbb{Z}$ such that $w_{1} \overline{w_{1}} \equiv 1(\bmod p)$.

We now use induction to show the following $\forall k \geqslant 1$,

$$
\begin{aligned}
\left(p, \overline{m_{k}}\right) & =1,\left(p, b_{k}\right)=1 \\
a_{k} & \equiv b_{k} M N_{1} x w_{2} \overline{w_{1}} d(k)(\bmod p) .
\end{aligned}
$$

We have $\gamma_{1}=\frac{a_{1}}{b_{1}}$, where $\left(a_{1}, b_{1}\right)=1$. So $\overline{m_{1}}=b_{1}$. By Equation (8) in [6], we have $Q_{2}=Y^{b_{1}}-\lambda_{1} X^{a_{1}}$, for some $\lambda_{1} \in K \backslash\{0\}$. Recall, $H_{i, j, t, x}=\left\{\left(\alpha^{a i}, \beta^{b j}\right) \mid b \equiv\right.$ $a x(\bmod t)\}$. So $\left(\alpha^{i}, \beta^{x j}\right) \in H_{i, j, t, x}$. Now, $\left(\alpha^{i}, \beta^{x j}\right) \cdot Q_{2}=\beta^{b_{1} x j} Y^{b_{1}}-\lambda_{1} \alpha^{a_{1} i} X^{a_{1}}$. Since $Q_{2}$ is an eigenfunction for $H_{i, j, t, x}$, we have

$$
\begin{aligned}
\beta^{b_{1} x j}=\alpha^{a_{1} i} & \Longrightarrow \frac{b_{1} x j w_{2}}{n}-\frac{a_{1} i w_{1}}{m} \in \mathbb{Z} \text { by Remark 6.0.5 } \\
& \Longrightarrow \frac{b_{1} x w_{2}}{N t}-\frac{a_{1} w_{1}}{M t} \in \mathbb{Z}
\end{aligned}
$$




$$
\begin{aligned}
& \Longrightarrow M N t \mid\left[b_{1} x M w_{2}-a_{1} N w_{1}\right] \\
& \Longrightarrow b_{1} M N_{1} x w_{2} \overline{w_{1}} \equiv a_{1}(\bmod p) \text { as } p \mid t .
\end{aligned}
$$

If $\left(p, b_{1}\right) \neq 1$, then $p\left|b_{1} \Longrightarrow p\right| a_{1}$. But this contradicts $\left(a_{1}, b_{1}\right)=1$. So, $\left(p, b_{1}\right)=1$. Since $\overline{m_{1}}=b_{1}$, we thus have $\left(p, \overline{m_{1}}\right)=1$. Thus we have the induction step for $k=1$. Suppose (6.2) is true for $k=1, \cdots, l-1$. From (5.2) we have $\left(\alpha^{a i}, \beta^{b j}\right) \cdot Q_{k}=$ $\beta^{d(k) b j} Q_{k} \forall k \geqslant 1, \forall\left(\alpha^{a i}, \beta^{b j}\right) \in H_{i, j, t, x}$. By Equation (8) in [6] we have, $Q_{l+1}=$ $Q_{l}^{\overline{m_{l}}}-\lambda_{l} X^{c_{0}} Y^{c_{1}} Q_{2}^{c_{2}} \cdots Q_{l-1}^{c_{l-1}}$ where $\lambda_{l} \in K \backslash\{0\}, 0 \leqslant c_{k}<\overline{m_{k}} \forall k=1, \cdots, l-1$ and $\overline{m_{l}} \gamma_{l}=\sum_{k=0}^{l-1} c_{k} \gamma_{k}$ $\left(\alpha^{i}, \beta^{x j}\right) \cdot Q_{l+1}=\beta^{x j \overline{m_{l}} d(l)} Q_{l}^{\overline{m_{l}}}-\lambda_{l} \alpha^{i c_{0}} \beta^{x j\left[\sum_{k=1}^{l-1} c_{k} d(k)\right]} X^{c_{0}} Y^{c_{1}} Q_{2}^{c_{2}} \cdots Q_{l-1}^{c_{l-1}}$. Since $Q_{l+1}$ is an eigenfunction for $H_{i, j, t, x}$, we have

$$
\begin{aligned}
& \beta^{x j \overline{m_{l}} d(l)}=\alpha^{i c_{0}} \beta^{x j\left[\sum_{k=1}^{l-1} c_{k} d(k)\right]} \\
\Longrightarrow & \beta^{x j\left[\overline{m_{l}} d(l)-\sum_{k=1}^{l-1} c_{k} d(k)\right]}=\alpha^{i c_{0}} \\
\Longrightarrow & \frac{x\left[\overline{m_{l}} d(l)-\sum_{k=1}^{l-1} c_{k} d(k)\right] w_{2}}{N t}-\frac{c_{0} w_{1}}{M t} \in \mathbb{Z} \text { by Remark } 6.0 .5 \\
\Longrightarrow & M N t \mid\left[M x w_{2} \overline{m_{l}} d(l)-M x w_{2} \sum_{k=1}^{l-1} c_{k} d(k)-N c_{0} w_{1}\right] \\
\Longrightarrow & p \mid\left[M x w_{2} \overline{m_{l}} d(l)-M x w_{2} \sum_{k=1}^{l-1} c_{k} d(k)-N c_{0} w_{1}\right] \\
\Longrightarrow & M N_{1} x w_{2} \overline{w_{1}} \overline{m_{l}} d(l) \equiv\left[M N_{1} x w_{2} \overline{w_{1}} \sum_{k=1}^{l-1} c_{k} d(k)+c_{0}\right](\bmod p) .
\end{aligned}
$$

Now, $p \mid \overline{m_{l}} \Longrightarrow c_{0}=\lambda p-M N_{1} x w_{2} \overline{w_{1}} \sum_{k=1}^{l-1} c_{k} d(k)$, where $\lambda \in \mathbb{Z}$. Let $\overline{m_{l}}=$ $p M_{l}$, where $M_{l} \in \mathbb{Z}_{>0}$. So, $\overline{m_{l}} \gamma_{l}=p M_{l} \gamma_{1}=c_{0}+\sum_{k=1}^{l-1} c_{k} \gamma_{k}=\lambda p+\sum_{k=1}^{l-1} c_{k}\left[\gamma_{k}-\right.$ $\left.M N_{1} x w_{2} \overline{w_{1}} d(k)\right]$. 
By our induction statement, $\forall k=1, \cdots, l-1$, we have $a_{k}=t_{k} p+b_{k} M N_{1} x w_{2} \overline{w_{1}} d(k)$, where $t_{k} \in \mathbb{Z}$. Thus,

$$
p M_{l} \gamma_{l}=\lambda p+\sum_{k=1}^{l-1} c_{k}\left[\frac{t_{k} p+b_{k} M N_{1} x w_{2} \overline{w_{1}} d(k)}{b_{k}}-M N_{1} x w_{2} \overline{w_{1}} d(k)\right]=\lambda p+p \sum_{k=1}^{l-1} c_{k} t_{k} \frac{1}{b_{k}} .
$$

Now $\left(a_{k}, b_{k}\right)=1 \Longrightarrow \exists h_{k} \in \mathbb{Z}$ such that $h_{k} a_{k} \equiv 1\left(\bmod b_{k}\right)$. Let $h_{k} a_{k}-1=\zeta_{k} b_{k}$, where $\zeta_{k} \in \mathbb{Z}$. So, $\frac{1}{b_{k}}=\frac{h_{k} a_{k}-\left(h_{k} a_{k}-1\right)}{b_{k}}=h_{k} \gamma_{k}-\zeta_{k}$. Then, $p M_{l} \gamma_{l}=\lambda p+p \sum_{k=1}^{l-1} c_{k} t_{k}\left[h_{k} \gamma_{k}-\zeta_{k}\right]$ implies

$$
M_{l} \gamma_{l}=\lambda+\sum_{k=1}^{l-1} c_{k} t_{k}\left[h_{k} \gamma_{k}-\zeta_{k}\right] \in G\left(\gamma_{0}, \cdots, \gamma_{l-1}\right)
$$

But this contradicts the minimality of $\overline{m_{l}}$. So $p \nmid \overline{m_{l}}$. So $\left(p, \overline{m_{l}}\right)=1$.

Now, $\overline{m_{l}} \gamma_{l}=c_{0}+\sum_{k=1}^{l-1} c_{k} \gamma_{k} \Longrightarrow \overline{m_{l}} \frac{a_{l}}{b_{l}}=c_{0}+\sum_{k=1}^{l-1} c_{k} \frac{a_{k}}{b_{k}} \Longrightarrow \overline{m_{l}} a_{l} \prod_{k=1}^{l-1} b_{k}=$ $c_{0} B+B \sum_{k=1}^{l-1} c_{k} \frac{a_{k}}{b_{k}}$, where $B=\prod_{k=1}^{l} b_{k}$. From the induction hypothesis, $\frac{a_{k}}{b_{k}} B=$ $\left[t_{k} p+b_{k} M N_{1} x w_{2} \overline{w_{1}} d(k)\right] \frac{B}{b_{k}}$. So,

$$
\begin{aligned}
\overline{m_{l}} a_{l} \prod_{k=1}^{l-1} b_{k}=c_{0} B+\sum_{k=1}^{l-1} c_{k}\left[t_{k} p+b_{k} M N_{1} x w_{2} \overline{w_{1}} d(k)\right] \frac{B}{b_{k}} \\
\Longrightarrow \overline{m_{l}} a_{l} \prod_{k=1}^{l-1} b_{k} \equiv\left[c_{0}+M N_{1} x w_{2} \overline{w_{1}} \sum_{k=1}^{l-1} c_{k} d(k)\right] B(\bmod p) .
\end{aligned}
$$

Since, $M N_{1} x w_{2} \overline{w_{1}} \overline{m_{l}} d(l) \equiv\left[M N_{1} x w_{2} \overline{w_{1}} \sum_{k=1}^{l-1} c_{k} d(k)+c_{0}\right](\bmod p)$, we have

$$
\overline{m_{l}} a_{l} \prod_{k=1}^{l-1} b_{k} \equiv M N_{1} x w_{2} \overline{w_{1}} \overline{m_{l}} d(l) \prod_{k=1}^{l} b_{k}(\bmod p)
$$

Since $\left(p, \overline{m_{l}}\right)=1,\left(p, b_{k}\right)=1 \forall k=1, \cdots, l-1$, we have $a_{l} \equiv M N_{1} x w_{2} \overline{w_{1}} d(l) b_{l}(\bmod p)$. If $p \mid b_{l}$, then $p \mid a_{l}$ which contradicts $\left(a_{l}, b_{l}\right)=1$. So $\left(p, b_{l}\right)=1$. Thus we have the induction step for $k=l$.

In particular, by induction we have $\left(p, \overline{m_{k}}\right)=1 \forall k \geqslant 1$. Since $d(k)=\overline{m_{1}} \cdots \overline{m_{k-1}}$ (by 2), Chapter 4), we have $(p, d(k))=1 \forall k \geqslant 2$. So $p \nmid d(k) \forall k \geqslant 2 \Longrightarrow t \nmid$ 
$d(k) \forall k \geqslant 2 \Longrightarrow \frac{n}{j}=N t \nmid d(k) \forall k \geqslant 2$. Thus by Lemma 6.0 .3 , we have $S^{R_{\mathfrak{m}}}(\nu)$ is not finitely generated over $S^{\left(A_{i, j, t, x}\right)_{\mathfrak{n}}}(\nu)$.

Proposition 6.0.7. Let $i, j, t, x$ be positive integers satisfying the conditions of Theorem 3.0.2, such that $\left(\frac{m}{i}, \frac{n}{j}\right)=t$ and $t>1$. Set $\frac{m}{i}=M t$ and $\frac{n}{j}=N t$ where $M, N \in \mathbb{Z}_{>0}$ and $(M, N)=1$. Suppose that for any prime number $p$ which divides $t$, the number $p$ also divides $N$. Suppose that $\nu$ is a rational rank 1 non discrete valuation dominating $R_{\mathfrak{m}}$ with a generating sequence (4.2) of eigenfunctions for $H_{i, j, t, x}$. Then $S^{R_{\mathfrak{m}}}(\nu)$ is not finitely generated over $S^{\left(A_{i, j, t, x}\right)_{\mathfrak{n}}}(\nu)$.

Proof. Since $(x, t)=1, \exists r \in \mathbb{Z}_{>0}$ such that $r x \equiv 1(\bmod t)$. So $(r, t)=1$. Recall, $\alpha=\delta^{w_{1} n}, \beta=\delta^{w_{2} m}$, where $\delta$ is a primitive $m n$-th root of unity, and $\left(w_{1}, m\right)=$ $1,\left(w_{2}, n\right)=1,1 \leqslant w_{1} \leqslant m$ and $1 \leqslant w_{2} \leqslant n$. Now, $M \mid m \Longrightarrow\left(w_{1}, M\right)=1$. Similarly, $\left(w_{2}, N\right)=1,\left(w_{1}, t\right)=1,\left(w_{2}, t\right)=1$. So $\exists \overline{w_{1}}, \overline{w_{2}} \in \mathbb{Z}_{>0}$ such that $w_{1} \overline{w_{1}} \equiv 1(\bmod t)$ and $w_{2} \overline{w_{2}} \equiv 1(\bmod t)$.

Write $N=\bar{N} N^{\prime}$, where $\bar{N}$ is the largest factor of $N$ such that $(\bar{N}, x)=1$. If $\bar{N}=1$, then for any prime $p$ dividing $N$, we have $p \mid x$. So in particular $p|t \Longrightarrow p| x$. But this is a contradiction as $(t, x)=1$. So $\bar{N}>1$ if $N>1$. We will now show $\left(\bar{N}, N^{\prime}\right)=1$. Suppose the contrary. Then $\exists$ a prime $p$ such that $p \mid \bar{N}$ and $p \mid N^{\prime}$. $p \mid \bar{N} \Longrightarrow(p, x)=1 \Longrightarrow(\bar{N} p, x)=1$. And, $\bar{N} N^{\prime}=N \Longrightarrow p \bar{N} \mid N$. This contradicts the maximality of $\bar{N}$. So $\left(\bar{N}, N^{\prime}\right)=1$. Hence $(N, x)=\left(N^{\prime}, x\right)$. We will now show that $\left(t, N^{\prime}\right)=1$. Suppose $\exists$ a prime $p$ such that $p \mid t$ and $p \mid N^{\prime}$. Then $p|t, p| N$ and $p \nmid \bar{N}$. Thus $p \mid t$ and $p \mid x$, which is a contradiction as $t$ and $x$ are coprime. Thus $\left(t, N^{\prime}\right)=1$. Also $\left(N, w_{2}\right)=1$ implies $\left(\bar{N}, w_{2}\right)=1$.

Let $\left\{Q_{l}\right\}_{l \geqslant 0}$ be the generating sequence (4.2) of the valuation $\nu$, where $Q_{0}=X, Q_{1}=$ 
$Y$, and each $Q_{l}$ is an eigenfunction for $H_{i, j, t, x}$. Let $\gamma_{l}=\nu\left(Q_{l}\right) \forall l \geqslant 0$. Without any loss of generality, we can assume $\gamma_{0}=1$. Let $\gamma_{1}=\frac{a_{1}}{b_{1}}$, where $\left(a_{1}, b_{1}\right)=1$. So $\overline{m_{1}}=b_{1}$. By Equation (8) in [6], we have $Q_{2}=Y^{b_{1}}-\zeta_{1} X^{a_{1}}$ for some $\zeta_{1} \in K \backslash\{0\}$. Now, $\left(\alpha^{i}, \beta^{x j}\right) \in H_{i, j, t, x}$. By (5.2), $\left(\alpha^{a i}, \beta^{b j}\right) \cdot Q_{k}=\beta^{d(k) b j} Q_{k} \forall k \geqslant 1, \forall\left(\alpha^{a i}, \beta^{b j}\right) \in$ $H_{i, j, t, x}$. Now,$\left(\alpha^{i}, \beta^{x j}\right) \cdot Q_{2}=\left(\alpha^{i}, \beta^{x j}\right) \cdot\left[Y^{b_{1}}-\zeta_{1} X^{a_{1}}\right]=\beta^{b_{1} x j} Y^{b_{1}}-\zeta_{1} \alpha^{a_{1} i} X^{a_{1}}$. Since $Q_{2}$ is an eigenfunction for $H_{i, j, t, x}$, we have

$$
\begin{aligned}
& \beta^{b_{1} x j}=\alpha^{a_{1} i} \Longrightarrow \frac{b_{1} x w_{2}}{N t}-\frac{a_{1} w_{1}}{M t} \in \mathbb{Z} \text { by Remark 6.0.5 } \\
& \Longrightarrow M \bar{N} t \mid\left[M b_{1} x w_{2}-N a_{1} w_{1}\right] \\
& \Longrightarrow M \mid a_{1} \text { and } \bar{N} \mid b_{1} \text { as }\left(\bar{N}, w_{2}\right)=1,\left(M, w_{1}\right)=1,(M, N)=1,(\bar{N}, x)=1 .
\end{aligned}
$$

Let $a_{1}=M a_{1}^{\prime}$ and $b_{1}=\bar{N} b_{1}^{\prime}$. Then, $M \bar{N} t \mid\left[M \bar{N} b_{1}^{\prime} x w_{2}-N M a_{1}^{\prime} w_{1}\right]$ implies $b_{1}^{\prime} \equiv$ $r a_{1}^{\prime} w_{1} \overline{w_{2}} N^{\prime}(\bmod t)$ as $r x \equiv 1(\bmod t)$ and $N=\bar{N} N^{\prime}$. Now, $\gamma_{1}=\frac{a_{1}}{b_{1}}=\frac{M a_{1}^{\prime}}{\bar{N} b_{1}^{\prime}} .\left(a_{1}, b_{1}\right)=$ $1 \Longrightarrow\left(\bar{N}, a_{1}^{\prime}\right)=1,\left(a_{1}^{\prime}, b_{1}^{\prime}\right)=1$ and $\left(M, b_{1}^{\prime}\right)=1$. Rename $a_{1}^{\prime}=u$ and $b_{1}^{\prime}=r^{\prime}$. Then $(u, \bar{N})=1$. If $(u, t) \neq 1$, then $\exists$ a prime $p$ such that $p \mid t$ and $p \mid u$. Thus $p|t, p| N$ and $p \nmid \bar{N}$, since for any prime $p$ dividing $t, p$ also divides $N$. So $p \mid t$ and $p \mid N^{\prime}$. But we have established earlier that $\left(t, N^{\prime}\right)=1$. So $(u, t)=1$. And, $r^{\prime} \equiv r u w_{1} \overline{w_{2}} N^{\prime}(\bmod t) \Longrightarrow r^{\prime} x \equiv u w_{1} \overline{w_{2}} N^{\prime}(\bmod t)$. Thus,

$$
\gamma_{1}=\frac{M u}{\bar{N} r^{\prime}} \text { where }(u, \bar{N})=1,(u, t)=1,\left(u, r^{\prime}\right)=1,\left(M, r^{\prime}\right)=1, r^{\prime} \equiv r u w_{1} \overline{w_{2}} N^{\prime}(\bmod t) \text {. }
$$

We will now use induction to show that $\forall k \geqslant 2$,

$$
\begin{aligned}
& \gamma_{k}=M u \overline{m_{2}} \cdots \overline{m_{k-1}}+\frac{M \bar{N} t \lambda_{k}}{\overline{m_{1}} \cdots \overline{m_{k}}} \text { for some } \lambda_{k} \in \mathbb{Z} \\
& \left(t, \overline{m_{k}}\right)=1 .
\end{aligned}
$$


By Equation (8) in [6] we have, $Q_{3}=Q_{2}^{\overline{m_{2}}}-\zeta_{2} X^{c_{0}} Y^{c_{1}}$ where $\zeta_{2} \in K \backslash\{0\}, c_{0} \in$ $\mathbb{Z}_{>0}, 0 \leqslant c_{1}<\overline{m_{1}} .\left(\alpha^{i}, \beta^{x j}\right) \cdot Q_{3}=\beta^{x j \overline{m_{2}} \overline{m_{1}}} Q_{2}^{\overline{m_{2}}}-\zeta_{2} \alpha^{i c_{0}} \beta^{x j c_{1}} X^{c_{0}} Y^{c_{1}}$. Since $Q_{3}$ is an eigenfunction for $H_{i, j, t, x}$, we have

$$
\begin{aligned}
& \beta^{x j \overline{m_{2}} \overline{m_{1}}}=\alpha^{i c_{0}} \beta^{x j c_{1}} \Longrightarrow \beta^{x j\left[\overline{m_{2}} \overline{m_{1}}-c_{1}\right]}=\alpha^{i c_{0}} \\
& \Longrightarrow \frac{x\left[\overline{m_{2}} \overline{m_{1}}-c_{1}\right] w_{2}}{N t}-\frac{c_{0} w_{1}}{M t} \in \mathbb{Z} \text { by Remark 6.0.5 } \\
& \Longrightarrow M \bar{N} t \mid\left[M \bar{N} r^{\prime} x w_{2} \overline{m_{2}}-M x w_{2} c_{1}-N c_{0} w_{1}\right] \text { as } \overline{m_{1}}=\bar{N} r^{\prime} \\
& \Longrightarrow M \mid c_{0} \text { and } \bar{N} \mid c_{1} \text { as }(M, N)=1,\left(M, w_{1}\right)=1,\left(\bar{N}, w_{2}\right)=1,(\bar{N}, x)=1 .
\end{aligned}
$$

Let $c_{0}=M c_{0}^{\prime}$ and $c_{1}=\bar{N} c_{1}^{\prime}$. Plugging them in the above expression and using (6.3), we obtain,

$$
\begin{aligned}
& M \bar{N} t \mid\left[M \bar{N} r^{\prime} x w_{2} \overline{m_{2}}-M x w_{2} \bar{N} c_{1}^{\prime}-N M c_{0}^{\prime} w_{1}\right] \\
& \Longrightarrow r^{\prime} x w_{2} \overline{m_{2}} \equiv\left[w_{1} c_{0}^{\prime} N^{\prime}+x w_{2} c_{1}^{\prime}\right](\bmod t) \\
& \Longrightarrow u w_{1} \overline{m_{2}} N^{\prime} \equiv\left[w_{1} c_{0}^{\prime} N^{\prime}+x w_{2} c_{1}^{\prime}\right](\bmod t) \\
& \Longrightarrow r^{\prime} u \overline{m_{2}} \equiv\left[r^{\prime} c_{0}^{\prime}+u c_{1}^{\prime}\right](\bmod t) .
\end{aligned}
$$

So, $\overline{m_{2}} \gamma_{2}=c_{0}+c_{1} \gamma_{1}=M c_{0}^{\prime}+\bar{N} c_{1}^{\prime} \frac{M u}{\bar{N} r^{\prime}}=M\left[\frac{c_{0}^{\prime} r^{\prime}+c_{1}^{\prime} u}{r^{\prime}}\right]=M\left[\frac{r^{\prime} u \overline{m_{2}}+\lambda_{2} t}{r^{\prime}}\right]=M u \overline{m_{2}}+\frac{M \bar{N} t \lambda_{2}}{\overline{m_{1}}}$ for some $\lambda_{2} \in \mathbb{Z}$. Thus, $\gamma_{2}=M u+\frac{M \bar{N} t \lambda_{2}}{\overline{m_{1}} \overline{m_{2}}}$.

We will now show $\left(t, \overline{m_{2}}\right)=1$. Suppose if possible $\exists$ a prime $p$ such that $p \mid t$ and $p \mid \overline{m_{2}}$. Let $\overline{m_{2}}=p M_{2}$. So, $\gamma_{2}=M u+\frac{M \bar{N} t \lambda_{2}}{\overline{m_{1}} \overline{m_{2}}} \Longrightarrow \overline{m_{2}} \gamma_{2}=M u \overline{m_{2}}+\frac{M \bar{N} t \lambda_{2}}{\overline{m_{1}}} \Longrightarrow$ $p M_{2} \gamma_{2}=p M u M_{2}+\frac{M t \lambda_{2}}{r^{\prime}} \Longrightarrow r^{\prime} M_{2} \gamma_{2}=r^{\prime} M u M_{2}+M \lambda_{2} \frac{t}{p}$. $\left(w_{1}, t\right)=1 . \quad\left(N^{\prime}, t\right)=1 . r x \equiv 1(\bmod t)$ implies $(r, t)=1 . w_{2} \overline{w_{2}} \equiv 1(\bmod t)$ implies 
$\left(\overline{w_{2}}, t\right)=1$. And, $(u, t)=1$ by $(6.3)$. So, $r^{\prime} \equiv r u w_{1} \overline{w_{2}} N^{\prime}(\bmod t) \Longrightarrow\left(r^{\prime}, t\right)=1$. So $\exists r_{1} \in \mathbb{Z}$ such that $r_{1} r^{\prime} \equiv 1(\bmod t)$. So in particular, $r_{1} r^{\prime} \equiv 1(\bmod p) \forall$ prime $p$ dividing $t$. We then have,

$$
\begin{aligned}
& r_{1} r^{\prime} M_{2} \gamma_{2}=r_{1} r^{\prime} M u M_{2}+r_{1} M \lambda_{2} \frac{t}{p} \\
& \Longrightarrow\left(1+\mu_{2} p\right) M_{2} \gamma_{2}=r_{1} r^{\prime} M u M_{2}+r_{1} M \lambda_{2} \frac{t}{p} \text { for some } \mu_{2} \in \mathbb{Z} \\
& \Longrightarrow M_{2} \gamma_{2}+\mu_{2} \overline{m_{2}} \gamma_{2} \in \mathbb{Z} \subset G\left(\gamma_{0}, \gamma_{1}\right) \Longrightarrow M_{2} \gamma_{2} \in G\left(\gamma_{0}, \gamma_{1}\right) .
\end{aligned}
$$

But this contradicts the minimality of $\overline{m_{2}}$. So for any prime $p$ dividing $t$, we have $p \nmid \overline{m_{2}}$. Thus $\left(t, \overline{m_{2}}\right)=1$. We now have the induction step for $k=2$.

Suppose (6.4) is true for $k=3, \cdots, l-1$. By Equation (8) in [6] we have, $Q_{l+1}=Q_{l}^{\overline{m_{l}}}-$ $\zeta_{l} X^{c_{0}} Y^{c_{1}} Q_{2}^{c_{2}} \cdots Q_{l-1}^{c_{l-1}}$ where $\zeta_{l} \in K \backslash\{0\}, c_{0} \in \mathbb{Z}_{>0}, 0 \leqslant c_{k}<\overline{m_{k}} \forall k=1, \cdots, l-1$ and $\overline{m_{l}} \gamma_{l}=\sum_{k=0}^{l-1} c_{k} \gamma_{k}$. By 2) of Chapter 4 we have $d(l)=\prod_{k=1}^{l-1} \overline{m_{k}} \forall l \geqslant 2$. Again, $\overline{m_{1}}=\bar{N} r^{\prime}$ by (6.3). So $\forall l \geqslant 2, d(l)=\bar{N} r^{\prime} \overline{d(l)}$, where $\overline{d(l)}=\frac{d(l)}{\overline{m_{1}}}$. Thus, $\forall l \geqslant 3, \overline{d(l)}=$ $\prod_{k=2}^{l-1} \overline{m_{k}}$

Now, $\left(\alpha^{i}, \beta^{x j}\right) \cdot Q_{l+1}=\beta^{x j \overline{m_{l}} d(l)} Q_{l}^{\overline{m_{l}}}-\zeta_{l} \alpha^{i c_{0}} \beta^{x j\left[\sum_{k=1}^{l-1} c_{k} d(k)\right]} X^{c_{0}} Y^{c_{1}} Q_{2}^{c_{2}} \cdots Q_{l-1}^{c_{l-1}}$. Since $Q_{l+1}$ is an eigenfunction for $H_{i, j, t, x}$ we have

$$
\begin{aligned}
& \beta^{x j\left[d(l+1)-\sum_{k=1}^{l-1} c_{k} d(k)\right]}=\alpha^{i c_{0}} \\
& \Longrightarrow \frac{x w_{2}\left[d(l+1)-\sum_{k=1}^{l-1} c_{k} d(k)\right]}{N t}-\frac{c_{0} w_{1}}{M t} \in \mathbb{Z} \text { by Remark 6.0.5 } \\
& \Longrightarrow M \bar{N} t \mid\left[M x w_{2} \bar{N} r^{\prime} \overline{d(l+1)}-M x w_{2} c_{1}-M x w_{2} \bar{N} r^{\prime} \sum_{k=2}^{l-1} c_{k} \overline{d(k)}-N c_{0} w_{1}\right] \\
& \Longrightarrow M \mid c_{0} \text { and } \bar{N} \mid c_{1} \text { as }(M, N)=1,\left(M, w_{1}\right)=1,(\bar{N}, x)=1,\left(\bar{N}, w_{2}\right)=1
\end{aligned}
$$

Let $c_{0}=M c_{0}^{\prime}$ and $c_{1}=\bar{N} c_{1}^{\prime}$. Plugging them in the above expression, and using (6.3), 
we obtain

$$
\begin{aligned}
& M \bar{N} t \mid\left[M x w_{2} \bar{N} r^{\prime} \overline{d(l+1)}-M x w_{2} \bar{N} c_{1}^{\prime}-M x w_{2} \bar{N} r^{\prime} \sum_{k=2}^{l-1} c_{k} \overline{d(k)}-N M w_{1} c_{0}^{\prime}\right] \\
& \Longrightarrow t \mid\left[x w_{2} r^{\prime} \overline{d(l+1)}-x w_{2} c_{1}^{\prime}-x w_{2} r^{\prime} \sum_{k=2}^{l-1} c_{k} \overline{d(k)}-w_{1} c_{0}^{\prime} N^{\prime}\right] \\
& \Longrightarrow r^{\prime} x w_{2} \overline{d(l+1)} \equiv\left[c_{0}^{\prime} w_{1} N^{\prime}+c_{1}^{\prime} x w_{2}+r^{\prime} x w_{2} \sum_{k=2}^{l-1} c_{k} \overline{d(k)}\right](\bmod t) \\
& \Longrightarrow r^{\prime} u \overline{d(l+1)} \equiv\left[r^{\prime} c_{0}^{\prime}+c_{1}^{\prime} u+r^{\prime} u \sum_{k=2}^{l-1} c_{k} \overline{d(k)}\right](\bmod t) .
\end{aligned}
$$

Now,

$$
\begin{aligned}
\overline{m_{l}} \gamma_{l} & =c_{0}+c_{1} \gamma_{1}+\sum_{k=2}^{l-1} c_{k} \gamma_{k} \\
& =M c_{0}^{\prime}+\bar{N} c_{1}^{\prime} \frac{M u}{\bar{N} r^{\prime}}+\sum_{k=2}^{l-1} c_{k}\left[M u \overline{d(k)}+\frac{M \bar{N} t \lambda_{k}}{d(k+1)}\right] \text { where } \lambda_{k} \in \mathbb{Z} \\
& =M\left[\frac{c_{0}^{\prime} r^{\prime}+c_{1}^{\prime} u+r^{\prime} u \sum_{k=2}^{l-1} c_{k} \overline{d(k)}}{r^{\prime}}+\frac{\bar{N} t \theta_{l}}{d(l)}\right] \text { for some } \theta_{l} \in \mathbb{Z} \\
& =M\left[\frac{r^{\prime} u \overline{d(l+1)}+\mu_{l} t}{r^{\prime}}+\frac{\bar{N} t \theta_{l}}{d(l)}\right] \text { for some } \mu_{l} \in \mathbb{Z} \\
& =M u \overline{d(l+1)}+\frac{M \bar{N} t \mu_{l}}{\overline{m_{1}}}+\frac{M \bar{N} t \theta_{l}}{d(l)} \\
& =M u \overline{d(l+1)}+\frac{M \bar{N} t \lambda_{l}}{d(l)} \text { for some } \lambda_{l} \in \mathbb{Z} \\
& \Longrightarrow \gamma_{l}=M u \overline{m_{2}} \ldots \overline{m_{l-1}}+\frac{M \bar{N} t \lambda_{l}}{\overline{m_{1}} \cdots \overline{m_{l}}}
\end{aligned}
$$

By our induction hypothesis, $\left(t, \overline{m_{k}}\right)=1 \forall k=2, \cdots, l-1$. So $\left(p, \overline{m_{k}}\right)=1$ for any prime $p$ dividing $t, \forall k=2, \cdots, l-1$, hence, $(p, \overline{d(l)})=1$. Suppose if possible $\exists$ a prime $p \mid t$ such that $p \mid \overline{m_{l}}$. Let $\overline{m_{l}}=p M_{l}$. Now, $\left(r^{\prime}, t\right)=1 \Longrightarrow\left(r^{\prime}, p\right)=1$. So $\left(p, r^{\prime} \overline{d(l)}\right)=1$. So $\exists r_{l} \in \mathbb{Z}$ such that $r_{l} r^{\prime} \overline{d(l)} \equiv 1(\bmod p)$. Let $r_{l} r^{\prime} \overline{d(l)}=1+\mu_{l} p$ for 
some $\mu_{l} \in \mathbb{Z}$. Now,

$$
\begin{aligned}
\gamma_{l} & =M u \overline{m_{2}} \cdots \overline{m_{l-1}}+\frac{M \bar{N} t \lambda_{l}}{\overline{m_{1}} \cdots \overline{m_{l}}} \\
& \Longrightarrow p M_{l} \gamma_{l}=M u \overline{m_{2}} \ldots \overline{m_{l}}+\frac{M t \lambda_{l}}{r^{\prime} \overline{d(l)}} \text { as } \overline{m_{l}}=p M_{l}, \overline{m_{1}}=\bar{N} r^{\prime}, \overline{d(l)}=\prod_{k=2}^{l-1} \overline{m_{k}} \\
& \Longrightarrow r^{\prime} \overline{d(l)} M_{l} \gamma_{l}=r^{\prime} \overline{d(l)} M u \overline{m_{2}} \cdots \overline{m_{l-1}} M_{l}+M \lambda_{l} \frac{t}{p} \text { as } \overline{m_{l}}=p M_{l} \\
& \Longrightarrow r_{l} r^{\prime} \overline{d(l)} M_{l} \gamma_{l}=r_{l} r^{\prime} \overline{d(l)} M u \overline{m_{2}} \cdots \overline{m_{l-1}} M_{l}+r_{l} M \lambda_{l} \frac{t}{p} \in \mathbb{Z} \\
& \Longrightarrow\left(1+\mu_{l} p\right) M_{l} \gamma_{l} \in \mathbb{Z} \Longrightarrow M_{l} \gamma_{l}+\mu_{l} \overline{m_{l}} \gamma_{l} \in \mathbb{Z} \subset G\left(\gamma_{0}, \cdots, \gamma_{l-1}\right) \\
& \Longrightarrow M_{l} \gamma_{l} \in G\left(\gamma_{0}, \cdots, \gamma_{l-1}\right) .
\end{aligned}
$$

But this contradicts the minimality of $\overline{m_{l}}$. So for any prime $p$ dividing $t$, we have $p \nmid \overline{m_{l}}$. Thus $\left(t, \overline{m_{l}}\right)=1$. We now have the induction step for $k=l$.

$$
\left(t, r^{\prime}\right)=1 \Longrightarrow \bar{N} t \nmid \bar{N} r^{\prime} \Longrightarrow N t \nmid \bar{N} r^{\prime} \Longrightarrow \frac{n}{j} \nmid \overline{m_{1}} \Longrightarrow \frac{n}{j} \nmid d(2) \text {. From the induction }
$$

we have $\left(t, \overline{m_{k}}\right)=1 \forall k \geqslant 2$. Thus $\left(t, \prod_{k=2}^{l-1} \overline{m_{k}}\right)=1 \Longrightarrow(t, \overline{d(l)})=1 \forall l \geqslant 3 \Longrightarrow$ $\left(t, r^{\prime} \overline{d(l)}\right)=1 \forall l \geqslant 3 . t \nmid r^{\prime} \overline{d(l)} \forall l \geqslant 3 \Longrightarrow \bar{N} t \nmid \bar{N} r^{\prime} \overline{d(l)} \forall l \geqslant 3 \Longrightarrow N t \nmid \overline{m_{1}} \overline{d(l)} \forall l \geqslant$ $3 \Longrightarrow \frac{n}{j} \nmid d(l) \forall l \geqslant 3$. So together we have, $\frac{n}{j} \nmid d(l) \forall l \geqslant 2$. Thus by Lemma 6.0.3, we have $S^{R_{\mathfrak{m}}}(\nu)$ is not finitely generated over $S^{\left(A_{i, j, t, x}\right)_{\mathfrak{n}}}(\nu)$.

We are now ready to prove Theorem 6.0.1.

Proof. Let $i, j, t, x$ be positive integers satisfying the conditions of Theorem 3.0.2 and suppose that $\nu$ is a rational rank 1 non discrete valuation dominating $R_{\mathfrak{m}}$ with a generating sequence (4.2) of eigenfunctions for $H_{i, j, t, x}$. By Proposition 6.0.4, we have $t \geqslant\left(\frac{m}{i}, \frac{n}{j}\right)$. Since $t \mid \frac{m}{i}$ and $t \mid \frac{n}{j}$, we have $\left(\frac{m}{i}, \frac{n}{j}\right)=t$.

Conversely, let $i, j, t, x$ be positive integers satisfying the conditions of Theorem 3.0.2 and suppose that $\left(\frac{m}{i}, \frac{n}{j}\right)=t$. We will show that $\exists$ a rational rank 1 non discrete 
valuation dominating $R_{\mathfrak{m}}$ with a generating sequence (4.2) of eigenfunctions for $H_{i, j, t, x}$. We consider the cases $t=1$ and $t>1$ separately.

Suppose that $\left(\frac{m}{i}, \frac{n}{j}\right)=t=1$. We will construct a rational rank 1 non discrete valuation $\nu$ dominating $R_{\mathfrak{m}}$, with a generating sequence (4.2) of eigenfunctions for $H_{i, j, t, x}$ such that $S^{R_{\mathfrak{m}}}(\nu)$ is finitely generated over $S^{\left(A_{i, j, t, x}\right)_{\mathfrak{n}}}(\nu)$. Let $\left\{q_{l}\right\}_{l \geqslant 2}$ be an infinite family of distinct prime numbers, such that $\left(q_{l}, \frac{m}{i}\right)=1,\left(q_{l}, \frac{n}{j}\right)=1$ for all $l \geqslant 2$. Let $q_{1}=\frac{n}{j}$. Let $\left\{c_{l}\right\}_{l \geqslant 1} \in \mathbb{Z}_{>0}$ be positive integers such that

$$
\begin{gathered}
c_{1}=\frac{m}{i}, c_{l} \equiv 0\left(\bmod \frac{m}{i}\right) \forall l \geqslant 1 \\
c_{l+1}>q_{l+1} c_{l} \forall l \geqslant 1,\left(c_{l}, q_{l}\right)=1 \forall l \geqslant 1 .
\end{gathered}
$$

We define a sequence of positive rational numbers $\left\{\gamma_{l}\right\}_{l \geqslant 0}$ as $\gamma_{0}=1, \gamma_{l}=\frac{c_{l}}{q_{l}} \forall l \geqslant 1$. We will show $\overline{m_{l}}=q_{l} \forall l \geqslant 1$, where $\overline{m_{l}}=\min \left\{q \in \mathbb{Z}_{>0} \mid q \gamma_{l} \in G\left(\gamma_{0}, \cdots, \gamma_{l-1}\right)\right\}$. Now, $\gamma_{1}=\frac{c_{1}}{q_{1}}=\frac{\left(\frac{m}{i}\right)}{\left(\frac{n}{j}\right)}$. Since $\left(\frac{m}{i}, \frac{n}{j}\right)=1$, we have $\overline{m_{1}}=\frac{n}{j}=q_{1}$. For $l \geqslant 2, q_{l} \gamma_{l}=c_{l} \in$ $\mathbb{Z} \Longrightarrow 1 \leqslant \overline{m_{l}} \leqslant q_{l}$. Suppose $q \in \mathbb{Z}_{>0}$ such that $q \gamma_{l}=q \frac{c_{l}}{q_{l}}=\sum_{k=0}^{l-1} a_{k} \gamma_{k}=\sum_{k=0}^{l-1} a_{k} \frac{c_{k}}{q_{k}}$. Then $q_{l} \mid q c_{l} \prod_{k=1}^{l-1} q_{k}$, that is, $q_{l} \mid q c_{l} \frac{n}{j} \prod_{k=2}^{l-1} q_{k}$. Now, $\left(q_{l}, c_{l}\right)=1$ and $\left(q_{l}, \frac{n}{j}\right)=1$. Again, $\left(q_{l}, q_{k}\right)=1 \forall k \neq l$, as they are distinct primes. So, $q_{l} \mid q$. Thus we have $\overline{m_{l}}=q_{l} \forall l \geqslant 1$. And, $\overline{m_{l}} \gamma_{l}=q_{l} \gamma_{l}=c_{l}<\frac{c_{l+1}}{q_{l+1}}=\gamma_{l+1}$. Thus we have a sequence of positive rational numbers $\left\{\gamma_{l}\right\}_{l \geqslant 0}$, such that $\gamma_{l+1}>\overline{m_{l}} \gamma_{l} \forall l \geqslant 1$. By Theorem 1.2 of [6], since $R_{\mathfrak{m}}$ is a regular local ring of dimension 2 , there is a valuation $\nu$ dominating $R_{\mathfrak{m}}$, such that $S^{R_{\mathfrak{m}}}(\nu)=S\left(\gamma_{0}, \gamma_{1}, \cdots\right) . \nu$ is a rational rank 1 non discrete valuation by the construction. By Theorem 4.2 of [6], $\exists$ a generating sequence (4.2) $\left\{Q_{l}\right\}_{l \geqslant 0}, Q_{0}=X, Q_{1}=Y, \cdots$ such that $\nu\left(Q_{l}\right)=\gamma_{l} \forall l \geqslant 0$.

From the recursive construction of the $\left\{\gamma_{l}\right\}_{l \geqslant 0}$, we have the generating sequence as 
$Q_{0}=X, Q_{1}=Y, Q_{2}=Y^{\frac{n}{j}}-\lambda_{1} X^{\frac{m}{i}}$, where $\lambda_{1} \in K \backslash\{0\}$. For all $l \geqslant 2, Q_{l+1}=$ $Q_{l}^{q_{l}}-\lambda_{l} X^{f_{0}} Y^{f_{1}} \cdots Q_{l-1}^{f_{l-1}}$, where $q_{l} \gamma_{l}=c_{l}=f_{0}+\sum_{k=1}^{l-1} f_{k} \gamma_{k}, 0 \leqslant f_{k}<\overline{m_{k}} \forall k \geqslant 1$. Now, $\left(c_{k}, q_{k}\right)=1 \forall k \geqslant 1$, and $\left(q_{k}, q_{h}\right)=1 \forall k \neq h$. So, $c_{l}=f_{0}+\sum_{k=1}^{l-1} \frac{f_{k} c_{k}}{q_{k}} \Longrightarrow c_{l} \prod_{k=1}^{l-1} q_{k}=$ $f_{0} \prod_{k=1}^{l-1} q_{k}+\frac{f_{1} c_{1} \prod_{k=1}^{l-1} q_{k}}{q_{1}}+\cdots+\frac{f_{l-1} c_{l-1} \prod_{k=1}^{l-1} q_{k}}{q_{l-1}}$, which implies $q_{k} \mid f_{k} \forall k \geqslant 1$. Since $0 \leqslant f_{k}<\overline{m_{k}}=q_{k}$, this implies $f_{k}=0 \forall k \geqslant 1$. So we have the generating sequence as,

$$
\begin{gathered}
Q_{0}=X, Q_{1}=Y, Q_{2}=Y^{\frac{n}{j}}-\lambda_{1} X^{\frac{m}{i}}, Q_{l+1}=Q_{l}^{q_{l}}-\lambda_{l} X^{c_{l}} \forall l \geqslant 2 \\
\text { where } \lambda_{l} \in K \backslash\{0\} \forall l \geqslant 1 .
\end{gathered}
$$

We now show that each $Q_{l}$ is an eigenfunction for $H_{i, j, 1,1} \cdot H_{i, j, 1,1}=\left\{\left(\alpha^{a i}, \beta^{b j}\right) \mid\right.$ $a, b \in \mathbb{Z}\}$. For all $l \geqslant 2, d(l)=\prod_{k=1}^{l-1} \overline{m_{k}}=q_{1} \cdots q_{l-1}=\frac{n}{j} q_{2} \cdots q_{l-1}$. We have, $\left(\alpha^{a i}, \beta^{b j}\right) \cdot Q_{2}=\beta^{b j \frac{n}{j}} Y^{\frac{n}{j}}-\lambda_{1} \alpha^{a i \frac{m}{i}} X^{\frac{m}{i}}=Q_{2}$. So, $Q_{2}$ is an eigenfunction. Suppose $Q_{3}, \cdots, Q_{l}$ are eigenfunctions for $H_{i, j, 1,1}$. We check for $Q_{l+1}$. From (5.2), $\left(\alpha^{a i}, \beta^{b j}\right)$. $Q_{k}=\beta^{b j d(k)} Q_{k} \forall 2 \leqslant k \leqslant l$. Since $\frac{m}{i} \mid c_{l}$ and $\frac{n}{j} \mid d(l)$, we have $\left(\alpha^{a i}, \beta^{b j}\right) \cdot Q_{l+1}=$ $\beta^{b j q_{l} d(l)} Q_{l}^{q_{l}}-\lambda_{l} \alpha^{a i c_{l}} X^{c_{l}}=Q_{l+1}$. Thus $Q_{l+1}$ is an eigenfunction. Thus by induction, $\left\{Q_{l}\right\}_{l \geqslant 0}$ is a generating sequence of eigenfunctions for $H_{i, j, 1,1}$.

Now we consider the case $\left(\frac{m}{i}, \frac{n}{j}\right)=t>1$. We will construct a rational rank 1 non discrete valuation $\nu$ dominating $R_{\mathfrak{m}}$, with a generating sequence (4.2) of eigenfunctions for $H_{i, j, t, x}$.

Since $(t, x)=1$, there are positive integers $r, s$ such that $r x-s t=1$. So $(r, t)=1$. From Lemma 3 in $\S 2$, Chapter $I I I$ of [12], we have that if $r, t$ are positive integers such that $(r, t)=1$, then there are infinitely many prime numbers of the form $r+\theta t$, where $\theta \in \mathbb{N}$. Define the family $\mathfrak{R}=\left\{r^{(k)}\right\}_{k \geqslant 0}$ as $r^{(0)}=r, r^{(k)}=k$-th prime in the above prime series. Any two elements in the family $\mathfrak{R}$ are coprime by construction. 
Also, $r^{(k)}=r+\theta_{k} t \Longrightarrow r^{(k)} \equiv r(\bmod t) \forall k$. Since $\mathfrak{R}$ is an infinite family such that any two elements in $\mathfrak{R}$ are mutually prime, it follows that there is an infinite ordered family of distinct prime numbers $\mathfrak{F}=\left\{r_{l}\right\}_{l \geqslant 1}$ such that, $r_{l} \equiv r(\bmod t),\left(r_{l}, \frac{\left(\frac{m}{i}\right)}{t}\right)=1$, $\left(r_{l}, \frac{\left(\frac{n}{j}\right)}{t}\right)=1,\left(r_{l}, w_{1}\right)=1,\left(r_{l}, w_{2}\right)=1 \forall l \geqslant 1$, where $w_{1}$ and $w_{2}$ are as in Remark 6.0.5. Let $d=\left(w_{1}, w_{2}\right)$. Thus $\left(\frac{w_{1}}{d}, \frac{w_{2}}{d}\right)=1$. Define two sequences $\left(a_{l}\right)_{l \geqslant 1}$ and $\left(b_{l}\right)_{l \geqslant 1}$ of non negative integers as follows,

$$
\begin{gathered}
b_{1}=0, r_{l}\left|b_{l} \forall l \geqslant 2, t\right| b_{l} \forall l \geqslant 2 \\
b_{l+1}>r_{l+1}\left[r^{l-1}+b_{l}\right]-r^{l} \forall l \geqslant 1 \\
a_{l}=\frac{\left(\frac{m}{i}\right)}{t}\left[r^{l-1}+b_{l}\right] \frac{w_{2}}{d} \forall l \geqslant 1 .
\end{gathered}
$$

Here $r_{l} \in \mathfrak{F} \forall l \geqslant 1$. Define a sequence of positive rational numbers $\left\{\gamma_{l}\right\}_{l \geqslant 0}$ as follows

$$
\begin{aligned}
\gamma_{0}=1, \gamma_{1} & =\frac{\frac{\left(\frac{m}{i}\right)}{t} \frac{w_{2}}{d}}{r_{1} \frac{\left(\frac{n}{j}\right)}{t} \frac{w_{1}}{d}}, \\
\gamma_{l} & =\frac{a_{l}}{r_{l}}=\frac{\left(\frac{m}{i}\right)}{t}\left[\frac{r^{l-1}+b_{l}}{r_{l}}\right] \frac{w_{2}}{d} \quad \forall l \geqslant 2 .
\end{aligned}
$$

We will show $\overline{m_{1}}=r_{1} \frac{\left(\frac{n}{j}\right)}{t} \frac{w_{1}}{d}$ and $\overline{m_{l}}=r_{l} \forall l \geqslant 2$, where $\overline{m_{l}}=\min \left\{q \in \mathbb{Z}_{>0} \mid q \gamma_{l} \in\right.$ $\left.G\left(\gamma_{0}, \cdots, \gamma_{l-1}\right)\right\} .\left(\frac{w_{1}}{d}, \frac{w_{2}}{d}\right)=1,\left(r_{1}, \frac{w_{2}}{d}\right)=1$ and $\left(\frac{\left(\frac{n}{j}\right)}{t}, \frac{w_{2}}{d}\right)=1$ implies $\left(\frac{w_{2}}{d}, r_{1} \frac{\left(\frac{n}{j}\right)}{t} \frac{w_{1}}{d}\right)=$ 1. Also, $\left(\frac{\left(\frac{m}{i}\right)}{t}, \frac{\left(\frac{n}{j}\right)}{t}\right)=1,\left(\frac{\left(\frac{m}{i}\right)}{t}, r_{1}\right)=1$ and $\left(\frac{\left(\frac{m}{i}\right)}{t}, \frac{w_{1}}{d}\right)=1$ implies $\left(\frac{\left(\frac{m}{i}\right)}{t}, r_{1} \frac{\left(\frac{n}{j}\right)}{t} \frac{w_{1}}{d}\right)=1$. Thus, $\left(\frac{w_{2}}{d} \frac{\left(\frac{m}{i}\right)}{t}, r_{1} \frac{\left(\frac{n}{j}\right)}{t} \frac{w_{1}}{d}\right)=1$, hence $\overline{m_{1}}=r_{1} \frac{\left(\frac{n}{j}\right)}{t} \frac{w_{1}}{d}$.

Now $\forall l \geqslant 2, r_{l} \gamma_{l}=a_{l} \in \mathbb{Z} \Longrightarrow 1 \leqslant \overline{m_{l}} \leqslant r_{l}$. Suppose $\exists$ a positive integer $q$ such that $q \gamma_{l} \in G\left(\gamma_{0}, \cdots, \gamma_{l-1}\right)$. Then $q \gamma_{l}=q \frac{a_{l}}{r_{l}}=c_{0}+c_{1} \frac{a_{1}}{r_{1} \frac{\left(\frac{n}{j}\right)}{t} \frac{w_{1}}{d}}+\sum_{k=2}^{l-1} c_{k} \frac{a_{k}}{r_{k}}$, where $c_{k} \in \mathbb{Z} \forall k=0, \cdots, l-1$. Thus $r_{l} \mid q a_{l} \frac{\left(\frac{n}{j}\right)}{t} \frac{w_{1}}{d} \prod_{k=1}^{l-1} r_{k} . \quad$ Now, $\left(r_{l}, \frac{\left(\frac{n}{j}\right)}{t}\right)=1$, and $\left(r_{l}, r_{k}\right)=1 \forall k \neq l$, as they are distinct primes. Also, $\left(r_{l}, \frac{w_{1}}{d}\right)=1$. So, $r_{l} \mid q a_{l}$. And, 
$r_{l}>r \Longrightarrow r_{l} \nmid r \Longrightarrow r_{l} \nmid \frac{\left(\frac{m}{i}\right)}{t}\left[r^{l-1}+b_{l}\right] \frac{w_{2}}{d}=a_{l}$ as $\left(r_{l}, \frac{w_{2}}{d}\right)=1,\left(r_{l}, \frac{\left(\frac{m}{i}\right)}{t}\right)=1$ and $r_{l} \mid b_{l}$.

Thus, $r_{l} \mid q$. Hence we have $\overline{m_{1}}=r_{1} \frac{\left(\frac{n}{j}\right)}{t} \frac{w_{1}}{d}$ and $\overline{m_{l}}=r_{l} \forall l \geqslant 2$.

Now, $b_{l+1}>r_{l+1}\left[r^{l-1}+b_{l}\right]-r^{l} \forall l \geqslant 1$ and $b_{1}=0$ implies $b_{2}>r_{2}-r$. Thus, $a_{2}=\frac{\left(\frac{m}{i}\right)}{t}\left[r+b_{2}\right] \frac{w_{2}}{d}>r_{2} \frac{\left(\frac{m}{i}\right)}{t} \frac{w_{2}}{d} \Longrightarrow \gamma_{2}=\frac{a_{2}}{r_{2}}>\frac{\left(\frac{m}{i}\right)}{t} \frac{w_{2}}{d}=\overline{m_{1}} \gamma_{1}$. For $l \geqslant 2$, we have $r^{l}+b_{l+1}>r_{l+1}\left[r^{l-1}+b_{l}\right] \Longrightarrow \frac{\left(\frac{m}{i}\right)}{t}\left[r^{l}+b_{l+1}\right] \frac{w_{2}}{d}>r_{l+1} \frac{\left(\frac{m}{i}\right)}{t}\left[r^{l-1}+b_{l}\right] \frac{w_{2}}{d} \Longrightarrow \gamma_{l+1}=\frac{a_{l+1}}{r_{l+1}}>$ $a_{l}=\overline{m_{l}} \gamma_{l}$.

Thus we have a sequence of positive rational numbers $\left\{\gamma_{l}\right\}_{l \geqslant 0}$ such that $\gamma_{l+1}>$ $\overline{m_{l}} \gamma_{l} \forall l \geqslant 1$. By Theorem 1.2 of [6], since $R_{\mathfrak{m}}$ is a regular local ring of dimension 2, there is a valuation $\nu$ dominating $R_{\mathfrak{m}}$, such that $S^{R_{\mathfrak{m}}}(\nu)=S\left(\gamma_{0}, \gamma_{1}, \cdots\right) . \quad \nu$ is a rational rank 1 non discrete valuation by the construction. By Theorem 4.2 of [6], $\exists$ a generating sequence (4.2) $\left\{Q_{l}\right\}_{l \geqslant 0}, Q_{0}=X, Q_{1}=Y, \cdots$ such that $\nu\left(Q_{l}\right)=\gamma_{l} \forall l \geqslant 0$. From the recursive construction of the $\left\{\gamma_{l}\right\}_{l \geqslant 0}$, we have the generating sequence as $Q_{0}=X, Q_{1}=Y, Q_{2}=Y^{r_{1} \frac{\left(\frac{n}{j}\right)}{t} \frac{w_{1}}{d}}-\lambda_{1} X^{\frac{\left(\frac{m}{i}\right)}{t} \frac{w_{2}}{d}}$. For all $l \geqslant 2, Q_{l+1}=Q_{l}^{r_{l}}-$ $\lambda_{l} X^{f_{0}} Y^{f_{1}} \cdots Q_{l-1}^{f_{l-1}}$, where $0 \leqslant f_{k}<\overline{m_{k}} \forall k \geqslant 1$ and $r_{l} \gamma_{l}=a_{l}=f_{0}+\sum_{k=1}^{l-1} f_{k} \gamma_{k}$. So, $a_{l}=f_{0}+\sum_{k=1}^{l-1} \frac{f_{k} a_{k}}{\overline{m_{k}}}$. We observe, from our construction, $\left(\overline{m_{k}}, \overline{m_{h}}\right)=1 \forall k \neq h$. Also, $\left(\overline{m_{k}}, a_{k}\right)=1 \forall k \geqslant 1$.

Thus, $a_{l} \prod_{k=1}^{l-1} \overline{m_{k}}=f_{0} \prod_{k=1}^{l-1} \overline{m_{k}}+\frac{f_{1} a_{1} \prod_{k=1}^{l-1} \overline{m_{k}}}{\overline{m_{1}}}+\cdots+\frac{f_{l-1} a_{l-1} \prod_{k=1}^{l-1} \overline{m_{k}}}{\overline{m_{l-1}}} \Longrightarrow \overline{m_{k}} \mid f_{k} \forall k \geqslant$ 1. Since $0 \leqslant f_{k}<\overline{m_{k}}$, we have $f_{k}=0 \forall k \geqslant 1$. Thus the generating sequence is given as,

$$
\begin{gathered}
Q_{0}=X, Q_{1}=Y, Q_{2}=Y^{r_{1} \frac{\left(\frac{n}{j}\right)}{t} \frac{w_{1}}{d}}-\lambda_{1} X^{\frac{\left(\frac{m}{i}\right)}{t} \frac{w_{2}}{d}} \\
Q_{l+1}=Q_{l}^{r_{l}}-\lambda_{l} X^{a_{l}} \forall l \geqslant 2 \\
\text { where } \lambda_{l} \in K \backslash\{0\} \forall l \geqslant 1 .
\end{gathered}
$$


This is a minimal generating sequence as $\overline{m_{l}}>1 \forall l \geqslant 1$. We now show that each $Q_{l}$ is an eigenfunction for $H_{i, j, t, x}$. From (4.1), $\left(\alpha^{a i}, \beta^{b j}\right) \cdot Q_{2}=\beta^{\frac{r_{1} b n}{t} \frac{w_{1}}{d}} Y^{r_{1} \frac{\left(\frac{n}{j}\right)}{t}} \frac{w_{1}}{d}-$ $\lambda_{1} \alpha^{\frac{a m}{t} \frac{w_{2}}{d}} X^{\frac{\left(\frac{m}{l}\right)}{t} \frac{w_{2}}{d}}$. Now, $\forall b \equiv a x(\bmod t), r_{1} b \equiv a(\bmod t)$, hence, $\left(\frac{r_{1} b-a}{t}\right)\left(\frac{w_{1} w_{2}}{d}\right) \in$ $\mathbb{Z}$. Thus by Remark 6.0.5, $\beta^{\frac{r_{1} b n}{t} \frac{w_{1}}{d}}=\alpha^{\frac{a m}{t} \frac{w_{2}}{d}} \forall b \equiv a x(\bmod t)$, that is, $Q_{2}$ is an eigenfunction for $H_{i, j, t, x}$.

Suppose $Q_{3}, \cdots, Q_{l}$ are eigenfunctions for $H_{i, j, t, x}$. We check for $Q_{l+1}$. We note $d(k)=$ $\overline{m_{1}} \cdots \overline{m_{k-1}}=\frac{\left(\frac{n}{j}\right)}{t} \frac{w_{1}}{d} r_{1} r_{2} \cdots r_{k-1}$. From (5.2) we have, $\left(\alpha^{a i}, \beta^{b j}\right) \cdot Q_{k}=\beta^{b j d(k)} Q_{k} \forall 1 \leqslant$ $k \leqslant l$. Now, $\left(\alpha^{a i}, \beta^{b j}\right) \cdot Q_{l+1}=\beta^{\frac{b n r_{1} \cdots r_{l}}{t} \frac{w_{1}}{d}} Q_{l}^{r_{l}}-\lambda_{l} \alpha^{a i a_{l}} X^{a_{l}}$. Since $r_{k} \equiv r(\bmod t) \forall k \geqslant 1$, $r x \equiv 1(\bmod t)$ and $t \mid b_{l}$, we have

$$
\begin{aligned}
& \frac{b r_{1} \cdots r_{l}}{t}-\frac{a r^{l-1}}{t} \in \mathbb{Z} \forall b \equiv a x(\bmod t) \\
\Longrightarrow & \frac{b r_{1} \cdots r_{l}}{t}-\frac{a\left[r^{l-1}+b_{l}\right]}{t} \in \mathbb{Z} \forall b \equiv a x(\bmod t) \\
\Longrightarrow & \frac{b r_{1} \cdots r_{l}}{t}\left(\frac{w_{1} w_{2}}{d}\right)-\frac{a\left[r^{l-1}+b_{l}\right]}{t}\left(\frac{w_{1} w_{2}}{d}\right) \in \mathbb{Z} \forall b \equiv a x(\bmod t) \\
\Longrightarrow & \frac{b n r_{1} \cdots r_{l}}{t}\left(\frac{w_{1} w_{2}}{d n}\right)-\frac{a i\left(\frac{m}{i}\right)\left[r^{l-1}+b_{l}\right]}{t}\left(\frac{w_{1} w_{2}}{d m}\right) \in \mathbb{Z} \forall b \equiv a x(\bmod t) \\
\Longrightarrow & \left(\frac{b n r_{1} \cdots r_{l}}{t} \frac{w_{1}}{d}\right) \frac{w_{2}}{n}-\left(a i a_{l}\right) \frac{w_{1}}{m} \in \mathbb{Z} \forall b \equiv a x(\bmod t) .
\end{aligned}
$$

Thus, by Remark 6.0.5, $\beta^{\frac{b n r_{1} \cdots r_{l}}{t} \frac{w_{1}}{d}}=\alpha^{a i a_{l}}$ for all $b \equiv a x(\bmod t)$, and hence $Q_{l+1}$ is an eigenfunction for $H_{i, j, t, x}$. Thus by induction, $\left\{Q_{l}\right\}_{l \geqslant 0}$ is a minimal generating sequence of eigenfunctions for $H_{i, j, t, x}$. This completes the proof of part 1) of Theorem 6.0 .1 .

Now we suppose $\left(\frac{m}{i}, \frac{n}{j}\right)=t=1$ and $\nu$ is a rational rank 1 non discrete valuation dominating $R_{\mathfrak{m}}$ with a generating sequence (4.2) of eigenfunctions for $H_{i, j, 1,1}$. Let $\nu\left(Q_{l}\right)=\gamma_{l} \forall l \in \mathbb{N}$. We have $Q_{0}=X, Q_{1}=Y$. By Equation (8) in [6], $Q_{2}=Y^{s}-\lambda X^{r}$ 
where $\lambda \in K \backslash\{0\}, s \gamma_{1}=r \gamma_{0}$. Since $\left(\frac{m}{i}, \frac{n}{j}\right)=1$, by Chinese Remainder Theorem (Theorem 2.1, $§ 2,[9])$ we have $H_{i, j, 1,1}$ is a cyclic group, generated by $\left(\alpha^{i}, \beta^{j}\right)$. By (4.1) we have $\left(\alpha^{i}, \beta^{j}\right) \cdot Q_{2}=\beta^{s j} Y^{s}-\lambda \alpha^{i r} X^{r}$. Since $Q_{2}$ is an eigenfunction, we have

$$
\begin{aligned}
\beta^{s j}=\alpha^{i r} & \Longrightarrow \frac{s j w_{2}}{n}-\frac{i r w_{1}}{m} \in \mathbb{Z} \text { by Remark 6.0.5 } \\
& \Longrightarrow \frac{s w_{2}}{\left(\frac{n}{j}\right)}-\frac{r w_{1}}{\left(\frac{m}{i}\right)} \in \mathbb{Z} \\
& \Longrightarrow \frac{m}{i} \mid r \text { and } \frac{n}{j} \mid s \text { as }\left(\frac{m}{i}, w_{1}\right)=1,\left(\frac{n}{j}, w_{2}\right)=1,\left(\frac{m}{i}, \frac{n}{j}\right)=1 .
\end{aligned}
$$

So, $Q_{2}=Y^{s}-\lambda X^{r} \in K\left[X^{\frac{m}{i}}, Y^{\frac{n}{j}}\right] \subset A_{i, j, 1,1}$. Thus by Proposition 6.0.2, we have part 2) of Theorem 6.0.1.

We observe that the part 3) of Theorem 6.0.1 follows from Propositions 6.0.6 and 6.0.7. This completes the proof of Theorem 6.0.1.

Corollary 6.0.8. Let $m>1$. Let $\left(c_{1}, m\right)=1$ and $\left(c_{2}, m\right)=1$. Let $\mathbb{U}_{m}$ acts on $R=K[X, Y]$ by the diagonal action given by $K$-algebra isomorphisms satisfying $\alpha \cdot X^{r} Y^{s}=\alpha^{c_{1} r+c_{2} s} X^{r} Y^{s}$. Suppose $\nu$ is a rational rank 1 non discrete valuation dominating $R_{\mathfrak{m}}$. Let $\left\{Q_{l}\right\}_{l \geqslant 0}$ be the generating sequence (4.2) of the valuation $\nu$, where $Q_{0}=X, Q_{1}=Y$, and suppose that each $Q_{l}$ is an eigenfunction for $\mathbb{U}_{m}$ under the diagonal action. Let $A=R^{\mathbb{U}_{m}}$ and $\mathfrak{a}=A \cap \mathfrak{m}$. Then $S^{R_{\mathfrak{m}}}(\nu)$ is not finitely generated over $S^{A_{\mathfrak{a}}}(\nu)$.

Proof. $\alpha$ is a primitive $m$-th root of unity, and $\left(c_{1}, m\right)=\left(c_{2}, m\right)=1$. So $\mathbb{U}_{m}=<\alpha>$ $=\left\langle\alpha^{c_{1}}>=\left\langle\alpha^{c_{2}}>\right.\right.$. The subgroup $H_{1,1, m, 1}$ of $\mathbb{U}_{m} \times \mathbb{U}_{m}$ is given by $H_{1,1, m, 1}=$ $\left\{\left(\left(\alpha^{c_{1}}\right)^{a},\left(\alpha^{c_{2}}\right)^{b}\right) \mid b \equiv a(\bmod m)\right\}=<\left(\alpha^{c_{1}}, \alpha^{c_{2}}\right)>$. From $(4.1)$, we have $H_{1,1, m, 1}$ acts on $R$ by $K$-algebra isomorphisms satisfying $\left(\alpha^{c_{1}}, \alpha^{c_{2}}\right) \cdot X^{r} Y^{s}=\alpha^{c_{1} r+c_{2} s} X^{r} Y^{s}$. Thus we have, $\alpha \cdot X^{r} Y^{s}=\left(\alpha^{c_{1}}, \alpha^{c_{2}}\right) \cdot X^{r} Y^{s}$. 
Now let $\left\{Q_{l}\right\}_{l \geqslant 0}$ be the generating sequence (4.2) of the valuation $\nu$, where $Q_{0}=$ $X, Q_{1}=Y$, and each $Q_{l}$ is an eigenfunction for $\mathbb{U}_{m}$ under the diagonal action. Hence each $Q_{l}$ is thus an eigenfunction for $H_{1,1, m, 1}$. And, $A=R^{\mathbb{U}_{m}}=R^{H_{1,1, m, 1}}=A_{1,1, m, 1}$. Also $\mathfrak{a}=A \cap \mathfrak{m}=A_{1,1, m, 1} \cap \mathfrak{m}=\mathfrak{n}$.

We now use the same notation as in Theorem 6.0.1. We have $i=1, j=1, t=m$. Since $m>1$, by Theorem 6.0.1 we have $S^{R_{\mathfrak{m}}}(\nu)$ is not finitely generated over $S^{\left(A_{1,1, m, 1}\right)_{\mathfrak{n}}}(\nu)$. Hence, $S^{R_{\mathfrak{m}}}(\nu)$ is not finitely generated over $S^{A_{\mathfrak{a}}}(\nu)$. 


\section{Chapter 7}

\section{Non-splitting}

Suppose that a local domain $B$ dominates a local domain $A$. Let $L$ be the quotient field of $A$ and $M$ be the quotient field of $B$. Suppose $\omega$ is a valuation of $L$ which dominates $A$. We say that $\omega$ does not split in $B$ if there is a unique extension $\omega^{*}$ of $\omega$ to $M$ which dominates $B$.

We use the same notation as in the previous chapters.

Theorem 7.0.1. Let $i, j, t, x$ be positive integers satisfying the conditions of Theorem 3.0.2 such that $\left(\frac{m}{i}, \frac{n}{j}\right)=t$. Suppose that $\nu$ is a rational rank 1 non discrete valuation dominating $R_{\mathfrak{m}}$ with a generating sequence (4.2) of eigenfunctions for $H_{i, j, t, x}$. Let $\bar{\nu}=\left.\nu\right|_{Q\left(A_{i, j, t, x}\right)}$ where $Q\left(A_{i, j, t, x}\right)$ denotes the quotient field of $A_{i, j, t, x}$. Then $\bar{\nu}$ does not split in $R_{\mathfrak{m}}$.

Proof. Let $\left\{Q_{k}\right\}_{k \geqslant 0},\left\{\gamma_{k}\right\}_{k \geqslant 0}$ and $\left\{\overline{m_{k}}\right\}_{k \geqslant 1}$ be as in Chapter 4. Thus $Q_{0}=X$ and $Q_{1}=Y$. Without any loss of generality, we can assume $\gamma_{0}=1$. Set $\frac{m}{i}=M t$ and $\frac{n}{j}=N t$ where $M, N \in \mathbb{Z}_{>0}$ and $(M, N)=1$. From (5.1) we have

$S^{\left(A_{i, j, t, x}\right)_{\mathfrak{n}}}(\nu)=\left\{\begin{array}{l|l}l \gamma_{0}+j_{1} \gamma_{1}+\cdots+j_{r} \gamma_{r} & \begin{array}{l}l \in \mathbb{N}, r \in \mathbb{N}, 0 \leqslant j_{k}<\overline{m_{k}} \forall k=1, \cdots, r \\ \alpha^{l a i} \beta^{b j} \sum_{k=1}^{r}\left[j_{k} d(k)\right]=1 \\ \forall b \equiv a x(\bmod t)\end{array}\end{array}\right.$.

Now, $\bar{\nu}=\left.\nu\right|_{Q\left(A_{i, j, t, x}\right)}$. Thus $S^{\left(A_{i, j, t, x}\right)_{\mathfrak{n}}}(\nu)=\left\{\nu(f) \mid 0 \neq f \in\left(A_{i, j, t, x}\right)_{\mathfrak{n}}\right\}=S^{\left(A_{i, j, t, x}\right)_{\mathfrak{n}}}(\bar{\nu})$.

The group generated by $S^{\left(A_{i, j, t, x}\right)_{\mathfrak{n}}}(\bar{\nu})$ is $\Gamma_{\bar{\nu}}$, the value group of $\bar{\nu}(1.2,[3])$. Thus $\Gamma_{\bar{\nu}}=$ 
$\left\{s_{1}-s_{2} \mid s_{1}, s_{2} \in S^{\left(A_{i, j, t, x}\right)_{\mathfrak{n}}}(\nu)\right\}$. Suppose $\gamma_{0} \in \Gamma_{\bar{\nu}}$. Then we have a representation,

$$
\gamma_{0}=\left(l_{1} \gamma_{0}+\sum_{k=1}^{r} h_{1, k} \gamma_{k}\right)-\left(l_{2} \gamma_{0}+\sum_{k=1}^{r} h_{2, k} \gamma_{k}\right)=\left(l_{1}-l_{2}\right) \gamma_{0}+\sum_{k=1}^{r}\left(h_{1, k}-h_{2, k}\right) \gamma_{k}
$$

where $l_{1} \gamma_{0}+\sum_{k=1}^{r} h_{1, k} \gamma_{k} \in S^{\left(A_{i, j, t, x}\right)_{\mathfrak{n}}}(\nu)$, and $l_{2} \gamma_{0}+\sum_{k=1}^{r} h_{2, k} \gamma_{k} \in S^{\left(A_{i, j, t, x}\right)_{\mathfrak{n}}}(\nu)$. Thus $l_{1}, l_{2} \in \mathbb{N}, r \in \mathbb{N}$ and $0 \leqslant h_{1, k}, h_{2, k}<\overline{m_{k}} \forall k=1, \cdots, r$. So, $\left|h_{1, k}-h_{2, k}\right|<\overline{m_{k}} \forall k=$ $1, \cdots, r$. Now $\left(h_{1, r}-h_{2, r}\right) \gamma_{r} \in G\left(\gamma_{0}, \cdots, \gamma_{r-1}\right)$ and $\left|h_{1, r}-h_{2, r}\right|<\overline{m_{r}} \Longrightarrow h_{1, r}=h_{2, r}$. With the same argument, we have $h_{1, k}=h_{2, k} \forall k=1, \cdots, r$. So in the representation of $\gamma_{0}$, we have $\gamma_{0}=\left(l_{1}-l_{2}\right) \gamma_{0} \Longrightarrow l_{1}-l_{2}=1$. Also,

$$
\begin{aligned}
& \alpha^{l_{1} a i} \beta^{b j \sum_{k=1}^{r}\left[h_{1, k} d(k)\right]}=1=\alpha^{l_{2} a i} \beta^{b j \sum_{k=1}^{r}\left[h_{2, k} d(k)\right]} \\
& \Longrightarrow \alpha^{\left(l_{1}-l_{2}\right) a i} \beta^{b j \sum_{k=1}^{r}\left[\left(h_{1, k}-h_{2, k}\right) d(k)\right]}=1 \forall b \equiv a x(\bmod t) .
\end{aligned}
$$

Since $l_{1}-l_{2}=1$ and $h_{1, k}=h_{2, k} \forall k=1, \cdots, r$, we have $\alpha^{a i}=1 \forall b \equiv a x(\bmod t)$. Thus $\alpha^{i}=1$, hence, $m \mid i$, that is, $m=i$. So we have obtained,

$$
\gamma_{0} \in \Gamma_{\bar{\nu}} \Longrightarrow M=1, t=1
$$

Suppose $\gamma_{1} \in \Gamma_{\bar{\nu}}$. Then we have a representation,

$$
\gamma_{1}=\left(l_{1} \gamma_{0}+\sum_{k=1}^{r} j_{1, k} \gamma_{k}\right)-\left(l_{2} \gamma_{0}+\sum_{k=1}^{r} j_{2, k} \gamma_{k}\right)=\left(l_{1}-l_{2}\right) \gamma_{0}+\sum_{k=1}^{r}\left(j_{1, k}-j_{2, k}\right) \gamma_{k}
$$

where $l_{1} \gamma_{0}+\sum_{k=1}^{r} j_{1, k} \gamma_{k} \in S^{\left(A_{i, j, t, x}\right)_{\mathfrak{n}}}(\nu)$, and $l_{2} \gamma_{0}+\sum_{k=1}^{r} j_{2, k} \gamma_{k} \in S^{\left(A_{i, j, t, x}\right)_{\mathfrak{n}}}(\nu)$. So, $l_{1}, l_{2} \in \mathbb{N}, r \in \mathbb{N}$ and $0 \leqslant j_{1, k}, j_{2, k}<\overline{m_{k}} \forall k=1, \cdots, r$. So, $\left|j_{1, k}-j_{2, k}\right|<\overline{m_{k}} \forall k=$ $1, \cdots, r$. Now, $\left(j_{1, r}-j_{2, r}\right) \gamma_{r} \in G\left(\gamma_{0}, \cdots, \gamma_{r-1}\right)$ and $\left|j_{1, r}-j_{2, r}\right|<\overline{m_{r}} \Longrightarrow j_{1, r}=j_{2, r}$. With the same argument, we have $j_{1, k}=j_{2, k} \forall k=2, \cdots r$. Thus we have, $\gamma_{1}=$ $\left(l_{1}-l_{2}\right) \gamma_{0}+\left(j_{1,1}-j_{2,1}\right) \gamma_{1}$ where $0 \leqslant\left|j_{1,1}-j_{2,1}\right|<\overline{m_{1}}$. Again, $\forall b \equiv a x(\bmod t)$ we 
have

$$
\alpha^{l_{1} a i} \beta^{b j \sum_{k=1}^{r}\left[j_{1, k} d(k)\right]}=1=\alpha^{l_{2} a i} \beta^{b j} \sum_{k=1}^{r}\left[j_{2, k} d(k)\right] .
$$

Since $d(1)=\operatorname{deg}_{Y}(Y)=1$ and $j_{1, k}=j_{2, k} \forall k=2, \cdots, r$, we have $\alpha^{\left(l_{1}-l_{2}\right) a i} \beta^{b j\left(j_{1,1}-j_{2,1}\right)}=$ 1 for all $b \equiv a x(\bmod t)$. So if $\gamma_{1} \in \Gamma_{\bar{\nu}}$, we have a representation

$$
\begin{aligned}
\gamma_{1}=l \gamma_{0}+j_{1} \gamma_{1} \text { where } l \in \mathbb{Z}, 0 \leqslant\left|j_{1}\right|<\overline{m_{1}} \\
\alpha^{l a i} \beta^{b j j_{1}}=1 \forall b \equiv a x(\bmod t) .
\end{aligned}
$$

In the above expression, $\left(1-j_{1}\right) \gamma_{1}=l \gamma_{0} \in \gamma_{0} \mathbb{Z} \Longrightarrow \overline{m_{1}} \mid\left(1-j_{1}\right)$.

And $\left|1-j_{1}\right| \leqslant 1+\left|j_{1}\right| \leqslant \overline{m_{1}} \Longrightarrow\left|1-j_{1}\right|=0$ or $\overline{m_{1}} .1-j_{1}=0 \Longrightarrow l=0, j_{1}=1$. From the above expression we then have, $\beta^{b j}=1 \forall b \equiv a x(\bmod t) \Longrightarrow n=j$. Now consider $\left|1-j_{1}\right|=\overline{m_{1}}$. If $1-j_{1}=-\overline{m_{1}}$ then $j_{1}=1+\overline{m_{1}}$ which contradicts $\left|j_{1}\right|<\overline{m_{1}}$. So $1-j_{1}=\overline{m_{1}}$, that is, $j_{1}=1-\overline{m_{1}}$. And $\left(1-j_{1}\right) \gamma_{1}=\overline{m_{1}} \gamma_{1}=l \gamma_{0}$. So $Q_{2}=Q_{1}^{\overline{m_{1}}}-\lambda X^{l}$ where $\lambda \in K \backslash\{0\} .\left(\alpha^{a i}, \beta^{b j}\right) \cdot Q_{2}=\beta^{b j \overline{m_{1}}} Q_{1}^{\overline{m_{1}}}-\lambda \alpha^{a i l} X^{l}$. Since $Q_{2}$ is an eigenfunction, we have $\beta^{b j \overline{m_{1}}}=\alpha^{a i l} \forall b \equiv a x(\bmod t)$. Again from the above expression we have, $\alpha^{a i l} \beta^{b j}=\beta^{b j \overline{m_{1}}} \forall b \equiv a x(\bmod t)$, as $j_{1}=1-\overline{m_{1}}$. Thus, $\beta^{b j}=1 \forall b \equiv a x(\bmod t)$, and hence $j=n$. So we have obtained,

$$
\gamma_{1} \in \Gamma_{\bar{\nu}} \Longrightarrow N=1, t=1
$$

For an element $g \in \Gamma_{\nu}$, let $[g]$ denote the class of $g$ in $\frac{\Gamma_{\nu}}{\Gamma_{\bar{\nu}}}$. Since $\frac{\Gamma_{\nu}}{\Gamma_{\bar{\nu}}}$ is a finite group, $[g]$ has finite order for each $g \in \Gamma_{\nu}$. Let $e=\left[\Gamma_{\nu}: \Gamma_{\bar{\nu}}\right]$.

First we suppose $\gamma_{0} \in \Gamma_{\bar{\nu}}$ and $\gamma_{1} \in \Gamma_{\bar{\nu}}$. From (7.1) and (7.2) we have $M=N=$ $t=1$. From Proposition 3.0.3 we have $\left|H_{i, j, t, x}\right|=M N t=1$. Thus, $M N t \mid e$.

Now we suppose $\gamma_{0} \notin \Gamma_{\bar{\nu}}$ and $\gamma_{1} \in \Gamma_{\bar{\nu}}$. From (7.2) we have $N=t=1$. From Proposition 3.0.3 we have $\left|H_{i, j, t, x}\right|=M N t=M$. Let $f_{0}$ denote the order of $\left[\gamma_{0}\right]$. 
Thus $f_{0} \gamma_{0} \in \Gamma_{\bar{\nu}}$. We thus have a representation

$$
f_{0} \gamma_{0}=\left(l_{1} \gamma_{0}+\sum_{k=1}^{r} h_{1, k} \gamma_{k}\right)-\left(l_{2} \gamma_{0}+\sum_{k=1}^{r} h_{2, k} \gamma_{k}\right)=\left(l_{1}-l_{2}\right) \gamma_{0}+\sum_{k=1}^{r}\left(h_{1, k}-h_{2, k}\right) \gamma_{k}
$$

where $l_{1} \gamma_{0}+\sum_{k=1}^{r} h_{1, k} \gamma_{k} \in S^{\left(A_{i, j, t, x}\right)_{\mathfrak{n}}}(\nu)$, and $l_{2} \gamma_{0}+\sum_{k=1}^{r} h_{2, k} \gamma_{k} \in S^{\left(A_{i, j, t, x}\right)_{\mathfrak{n}}}(\nu)$. Thus $l_{1}, l_{2} \in \mathbb{N}, r \in \mathbb{N}$ and $0 \leqslant h_{1, k}, h_{2, k}<\overline{m_{k}} \forall k=1, \cdots, r$. So, $\left|h_{1, k}-h_{2, k}\right|<\overline{m_{k}} \forall k=$ $1, \cdots, r$. With the same arguments as above, we have $h_{1, k}=h_{2, k} \forall k=1, \cdots, r$. Thus $f_{0} \gamma_{0}=\left(l_{1}-l_{2}\right) \gamma_{0} \Longrightarrow f_{0}=l_{1}-l_{2}$. And, for all $b \equiv a x(\bmod t)$,

$$
\alpha^{l_{1} a i} \beta^{b j} \sum_{k=1}^{r}\left[h_{1, k} d(k)\right]=1=\alpha^{l_{2} a i} \beta^{b j \sum_{k=1}^{r}\left[h_{2, k} d(k)\right]} .
$$

So, $\alpha^{\left(l_{1}-l_{2}\right) i}=\alpha^{f_{0} i}=1$, hence $M t\left|f_{0} \Longrightarrow M t\right| e$. Thus $M N t \mid e$ as $M N t=M$.

Now we suppose $\gamma_{0} \in \Gamma_{\bar{\nu}}$ and $\gamma_{1} \notin \Gamma_{\bar{\nu}}$. From (7.1) we have $M=t=1$. $\left|H_{i, j, t, x}\right|=M N t=N$. Let $f_{1}$ denote the order of $\left[\gamma_{1}\right]$, that is $f_{1} \gamma_{1} \in \Gamma_{\bar{\nu}}$. We have a representation,

$$
f_{1} \gamma_{1}=\left(l_{1} \gamma_{0}+\sum_{k=1}^{r} j_{1, k} \gamma_{k}\right)-\left(l_{2} \gamma_{0}+\sum_{k=1}^{r} j_{2, k} \gamma_{k}\right)=\left(l_{1}-l_{2}\right) \gamma_{0}+\sum_{k=1}^{r}\left(j_{1, k}-j_{2, k}\right) \gamma_{k}
$$

where $l_{1} \gamma_{0}+\sum_{k=1}^{r} j_{1, k} \gamma_{k} \in S^{\left(A_{i, j, t, x}\right)_{\mathfrak{n}}}(\nu)$, and $l_{2} \gamma_{0}+\sum_{k=1}^{r} j_{2, k} \gamma_{k} \in S^{\left(A_{i, j, t, x}\right)_{\mathfrak{n}}}(\nu)$. So, $l_{1}, l_{2} \in \mathbb{N}, r \in \mathbb{N}$ and $0 \leqslant j_{1, k}, j_{2, k}<\overline{m_{k}} \forall k=1, \cdots, r$. So, $\left|j_{1, k}-j_{2, k}\right|<\overline{m_{k}} \forall k=$ $1, \cdots, r$. With the same arguments as above, we have $j_{1, k}=j_{2, k} \forall k=2, \cdots, r$. So in the above representation, we have $f_{1} \gamma_{1}=\left(l_{1}-l_{2}\right) \gamma_{0}+\left(j_{1,1}-j_{2,1}\right) \gamma_{1}$ where $0 \leqslant\left|j_{1,1}-j_{2,1}\right|<\overline{m_{1}}$. Again, $\forall b \equiv a x(\bmod t)$ we have

$$
\alpha^{l_{1} a i} \beta^{b j \sum_{k=1}^{r}\left[j_{1, k} d(k)\right]}=1=\alpha^{l_{2} a i} \beta^{b j \sum_{k=1}^{r}\left[j_{2, k} d(k)\right]} .
$$

Since $d(1)=1$ and $j_{1, k}=j_{2, k} \forall k=2, \cdots, r$, we have $\alpha^{\left(l_{1}-l_{2}\right) a i} \beta^{b j\left(j_{1,1}-j_{2,1}\right)}=1$ for all $b \equiv a x(\bmod t)$. So we have a representation,

$$
f_{1} \gamma_{1}=l \gamma_{0}+j_{1} \gamma_{1} \text { where } l \in \mathbb{Z}, 0 \leqslant\left|j_{1}\right|<\overline{m_{1}}
$$

$$
\alpha^{l a i} \beta^{b j j_{1}}=1 \forall b \equiv a x(\bmod t)
$$


$\left(f_{1}-j_{1}\right) \gamma_{1}=l \gamma_{0} \Longrightarrow \overline{m_{1}} \mid\left(f_{1}-j_{1}\right)$. Let $f_{1}-j_{1}=c \overline{m_{1}}$ where $c \in \mathbb{Z}$. Let $\overline{m_{1}} \gamma_{1}=s \gamma_{0}$ where $s \in \mathbb{Z}_{>0}$. Thus $f_{1} \gamma_{1}=c s \gamma_{0}+j_{1} \gamma_{1} \Longrightarrow l \gamma_{0}=c s \gamma_{0}$. Thus $l=c s$. Since $\overline{m_{1}} \gamma_{1}=$ $s \gamma_{0}$, we have $Q_{2}=Q_{1}^{\overline{m_{1}}}-\lambda X^{s}$ where $\lambda \in K \backslash\{0\} .\left(\alpha^{a i}, \beta^{b j}\right) \cdot Q_{2}=\beta^{b j \overline{m_{1}}} Q_{1}^{\overline{m_{1}}}-\lambda \alpha^{a i s} X^{s}$. Since $Q_{2}$ is an eigenfunction we have, $\beta^{b j \overline{m_{1}}}=\alpha^{a i s} \forall b \equiv a x(\bmod t)$. Again, from the above expression of $f_{1} \gamma_{1}$, we have

$$
\begin{aligned}
& \alpha^{l a i} \beta^{b j\left(f_{1}-c \overline{m_{1}}\right)}=1 \forall b \equiv a x(\bmod t) \\
& \Longrightarrow \alpha^{c s a i} \beta^{b j f_{1}}=\beta^{b j \overline{m_{1}}} \forall b \equiv a x(\bmod t) \text { as } l=c s \\
& \Longrightarrow \beta^{b j f_{1}}=1 \forall b \equiv a x(\bmod t) \Longrightarrow N t\left|f_{1} \Longrightarrow N t\right| e .
\end{aligned}
$$

Thus we have obtained, $M N t \mid e$ as $M N t=N$.

Now we consider the final case, $\gamma_{0} \notin \Gamma_{\bar{\nu}}$ and $\gamma_{1} \notin \Gamma_{\bar{\nu}}$. Let $f_{0}$ denote the order of $\left[\gamma_{0}\right]$ and $f_{1}$ denote the order of $\left[\gamma_{1}\right]$ in $\frac{\Gamma_{\nu}}{\Gamma_{\bar{\nu}}}$. With the same arguments as before, we obtain $M t \mid f_{0}$ and $N t \mid f_{1}$. Thus we have $M t \mid e$ and $N t \mid e$. Now $(M t, N t)=t$. So the lowest common multiple of $M t$ and $N t$ is $\frac{M t N t}{t}=M N t$. Thus, $M N t \mid e$.

Now, $K(X, Y)$ is a Galois extension of $Q\left(A_{i, j, t, x}\right)$ with Galois group $H_{i, j, t, x}$ (Proposition 1.1.1, [2]). Thus $\left[K(X, Y): Q\left(A_{i, j, t, x}\right)\right]=\left|H_{i, j, t, x}\right|=M N t$ from Proposition 3.0.3. Let $\nu=\nu_{1}, \nu_{2}, \cdots, \nu_{r}$ be all the distinct extensions of $\bar{\nu}$ to $K(X, Y)$. Then (§12, Theorem 24, Corollary, [16]),

$$
\text { efr }=\left[K(X, Y): Q\left(A_{i, j, t, x}\right)\right]=M N t .
$$

Since $M N t \mid e$, we have $e=M N t, r=1$. So $\nu$ is the unique extension of $\bar{\nu}$ to $K(X, Y)$. Thus $\bar{\nu}$ does not split in $R_{\mathfrak{m}}$. 


\section{Bibliography}

[1] S. Abhyankar, On the valuations centered in a local domain, Amer. J. Math. 78 (1956), 321 - 348.

[2] D.J. Benson, Polynomial Invariants of Finite Groups, Cambridge University Press, 1993.

[3] S.D. Cutkosky, Ramification of valuations and local rings in positive characteristic, Communications in Algebra 44 (2016), 2828-2866.

[4] S.D. Cutkosky, Finite generation of extensions of associated graded rings along a valuation, to appear in the Journal of the London Math. Soc.

[5] S.D. Cutkosky, The role of defect and splitting in finite generation of extensions of associated graded rings along a valuation, Algebra and Number Theory 11 92017), 1461 - 1488.

[6] S.D. Cutkosky and Pham An Vinh, Valuation semigroups of two dimensional local rings, Proceedings of the London Mathematical Society 108 (2014), $350-384$.

[7] O. Kashcheyeva, Constructing examples of semigroups of valuations, J. Pure Appl. Algebra 200 (2016), 3826 - 3860.

[8] F.-V. Kuhlmann, Valuation theoretic and model theoretic aspects of local uniformization, in Resolution of Singularities - A Research Textbook in Tribute to Oscar Zariski, H. Hauser, J. Lipman, F. Oort, A. Quiros (es.), Progress in Math. 181, Birkhäuser (2000), 4559 - 4600.

[9] S. Lang, Algebra, revised third ed., Addison-Wesley Publishing Company Advanced Book Program, Reading, MA, 2002.

[10] M. Moghaddam, A construction for a class of valuations of the field $K\left(X_{1}, \ldots, X_{d}, Y\right)$ with large value group, Journal of Algebra, 319, 7 (2008), 2803-2829.

[11] J. Novacoski and M. Spivakovsky, Key polynomials and pseudo-convergent sequences, J. Algebra 495 (2018), 199 - 219.

[12] Jean-Pierre Serre, A Course In Arithmetic, Graduate Texts In Mathematics, 7, New York - Heidelberg - Berlin, Springer-Verlag, 1973. 
[13] M. Spivakovsky, Valuations in function fields of surfaces, Amer. J. Math. 112 (1990), $107-156$.

[14] B. Teissier, Valuations, deformations and toric geometry, Valuation theory and its applications II, F.V. Kuhlmann, S. Kuhlmann and M. Marshall, editors, Fields Institute Communications 33 (2003), Amer. Math. Soc., Providence, RI, $361-459$.

[15] B. Teissier, Overweight deformations of affine toric varieties and local uniformization, in Valuation theory in interaction, Proceedings of the second international conference on valuation theory, Segovia-El Escorial, 2011. Edited by A. Campillo, F-V- Kehlmann and B. Teissier. European Math. Soc. Publishing House, Congress Reports Series, Sept. 2014, 474 - 565.

[16] O. Zariski and P. Samuel, Commutative Algebra, Volume II, Van Nostrand, 1960. 


\section{VITA}

Arpan Dutta was born in Kolkata, India to Swapan Dutta and Ajanta Dutta in 1988. He obtained his B.Sc. in Mathematics and Computer Science from Chennai Mathematical Institute, Chennai, India in 2010 and his M.Sc. in Mathematics under the supervision of Dr. S. Senthamarai Kannan from Chennai Mathematical Institute, Chennai, India in 2012. Currently he is pursuing his Ph.D. from the University of Missouri-Columbia under the tutelage of Dr. Dale Cutkosky. For his Ph.D. he is studying generating sequences and semigroups of valuations on 2-dimensional normal local rings. 